\title{
Plane wave approximation of homogeneous Helmholtz solutions
}

\section{Journal Article}

\section{Author(s):}

Moiola, Andrea; Hiptmair, Ralf; Perugia, I.

Publication date:

2011-10

Permanent link:

https://doi.org/10.3929/ethz-b-000159705

Rights / license:

In Copyright - Non-Commercial Use Permitted

Originally published in:

Zeitschrift für angewandte Mathematik und Physik 62(5), https://doi.org/10.1007/s00033-011-0147-y 


\title{
Plane wave approximation of homogeneous Helmholtz solutions
}

\author{
A. Moiola, R. Hiptmair and I. Perugia
}

\begin{abstract}
In this paper, we study the approximation of solutions of the homogeneous Helmholtz equation $\Delta u+\omega^{2} u=0$ by linear combinations of plane waves with different directions. We combine approximation estimates for homogeneous Helmholtz solutions by generalized harmonic polynomials, obtained from Vekua's theory, with estimates for the approximation of generalized harmonic polynomials by plane waves. The latter is the focus of this paper. We establish best approximation error estimates in Sobolev norms, which are explicit in terms of the degree of the generalized polynomial to be approximated, the domain size, and the number of plane waves used in the approximations.
\end{abstract}

Mathematics Subject Classification (2010). 35J05 - 41A25 - 41A30.

Keywords. Approximation by plane waves . Generalized harmonic polynomials · Homogeneous Helmholtz solutions . Vekua's theory · Jacobi-Anger formula.

\section{Introduction}

This article is motivated by the recent surge in interest in numerical methods employing non-polynomial trial spaces for solutions of wave propagation problems. We focus our attention on the homogeneous Helmholtz equation $\Delta u+\omega^{2} u=0$ in $\mathbb{R}^{N}$ with constant coefficients and wave number $\omega>0$. In this context, a popular choice is to approximate $u$ locally or globally in spaces spanned by plane wave functions with different directions ${ }^{1} \boldsymbol{d}_{l} \in \mathbb{S}^{N-1}, l=1, \ldots, p$,

$$
P W_{\omega, p}\left(\mathbb{R}^{N}\right):=\left\{u \in C^{\infty}\left(\mathbb{R}^{N}\right): u(\boldsymbol{x})=\sum_{k=1}^{p} \alpha_{l} \mathrm{e}^{i \omega \boldsymbol{x} \cdot \boldsymbol{d}_{l}}, \alpha_{l} \in \mathbb{C}\right\}, \quad p \in \mathbb{N} .
$$

Examples of such numerical methods are the Plane Wave Partition of Unity Method (PW-PUM; see [1]), the Ultra Weak Variational Formulation (UWVF; see [5]), the Discontinuous Enrichment Method (DEM; see [7]) and the Plane Wave Discontinuous Galerkin Method (PWDG; see [4,11,13]), which generalizes the UWVF.

Numerical analysis of these methods often manages to establish quasi-optimality in the sense that the discretization error is closely linked to the best approximation error for $u$ in the trial spaces. Thus, convergence results for plane wave based approaches require best approximation estimates in Sobolev norms for homogeneous Helmholtz solutions by plane waves which are explicit in terms of the mesh size $h$ ( $h$-version) and in the number $p$ of plane waves within each element in the approximating spaces ( $p$-version).

Our objective is to derive approximation estimates of the form

$$
\inf _{w \in P W_{\omega, p}\left(\mathbb{R}^{N}\right)}\|u-w\|_{j, \omega, D} \leq \varepsilon\|u\|_{k, \omega, D} \quad \forall u \in H^{k}(D), \Delta u+\omega^{2} u=0 \quad \text { in } D,
$$

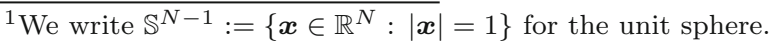


for $0 \leq j<k$, where $D \subset \mathbb{R}^{N}, N=2,3$, is a bounded domain and the wavenumber weighted norms are

$$
\|u\|_{k, \omega, \Omega}^{2}=\sum_{j=0}^{k} \omega^{2(k-j)}|u|_{j, \Omega}^{2} .
$$

Of course, in (1), we will establish the dependence of $\varepsilon$ on the size and the geometry of $D$, the number $p$ of directions $\boldsymbol{d}_{k}$ of plane waves, the regularity indices $j$ and $k$ as explicitly as possible. Moreover, as illustrated by the bound in (1), our principal interest is in the case of limited smoothness of $u$.

To tackle (1), we take a detour via spaces of so-called generalized harmonic polynomials

$$
H P_{\omega, L}\left(\mathbb{R}^{N}\right):= \begin{cases}\operatorname{span}\left\{\boldsymbol{x} \mapsto \mathrm{e}^{i l \psi} J_{|l|}(\omega r)\right\}_{l=-L}^{L} & \text { for } N=2, \\ \operatorname{span}\left\{\boldsymbol{x} \mapsto Y_{l, m}\left(\frac{\boldsymbol{x}}{|\boldsymbol{x}|}\right) j_{l}(\omega|\boldsymbol{x}|)\right\}_{\substack{l=0, \ldots, L \\ m=-l, \ldots, l}} & \text { for } N=3,\end{cases}
$$

where we used polar coordinates $(r, \psi)$ in two-dimensions. ${ }^{2}$ Generalized harmonic polynomials owe their pivotal role to Vekua's theory [23]. It supplies so-called Vekua operators, integral operators that map harmonic functions to solutions of the homogeneous Helmholtz equation and vice versa. In particular, they take harmonic polynomials to generalized harmonic polynomials. Using the continuity of Vekua operators [18], approximation estimates for homogeneous Helmholtz solutions in the spaces $H P_{\omega, L}\left(\mathbb{R}^{N}\right)$ can be obtained from approximation estimates of harmonic functions by harmonic polynomials. In [12], we have proved $h$-version approximation estimates for harmonic functions by harmonic polynomials in any space dimension, using a simple Bramble-Hilbert argument. Sharp two-dimensional $p$-estimates were proved in [16], heavily relying on complex analysis techniques. For the $p$-estimates in higher space dimensions, relying on the result of [2], in [12], we have proved algebraic convergence, but with order of convergence depending on the shape of the domain in an unknown way. All these results are reviewed in Sect. 3.

By introducing generalized harmonic polynomials, the task apparently reduces to estimating how well they can be approximated by plane waves:

$$
\inf _{w \in P W_{\omega, p}\left(\mathbb{R}^{N}\right)}\|u-w\|_{j, \omega, D} \leq\|u-Q\|_{j, \omega, D}+\inf _{w \in P W_{\omega, p}\left(\mathbb{R}^{N}\right)}\|Q-w\|_{j, \omega, D},
$$

for some judiciously chosen $Q \in H P_{\omega, L}\left(\mathbb{R}^{N}\right)$, which is "close" to $u$. Our chief target is to estimate the second term. In order to do this, in Sect. 4 , we prove algebraic orders of convergence in $h$ and more than exponential speed in $p$, the number of plane waves used in the approximation. The argument is based on the truncation and the inversion of the Jacobi-Anger expansion. In two space dimensions, any choice of propagation directions for the plane waves used in the approximation is allowed, while in three space dimensions, we ask a mild requirement for the $h$-convergence and a much stronger one for the $p$-convergence.

However, we eventually have to arrive at bounds in terms of $u$, which entails scrutinizing the link between $u$ and $Q$ in (3). This link is provided by Vekua's theory, and hence, we cannot avoid delving into it. In Sect. 5, we will combine all the results obtained or reported in the previous sections and write the final best approximation estimates for homogeneous Helmholtz solutions by plane waves (see Theorems 5.2 and 5.3 and Corollary 5.5).

\section{Vekua's theory}

In this section, we briefly summarize the main results concerning Vekua's theory and the generalized harmonic polynomials proved in $[12,18]$.

We will always consider a domain that satisfies the following assumption.

\footnotetext{
${ }^{2}$ In (2), we adopt the standard notation: $J_{l}$ stands for the Bessel functions of the first kind, $j_{l}$ designates the spherical Bessel functions and $Y_{l, m}$ the spherical harmonics.
} 
Assumption 2.1. Let $D \subset \mathbb{R}^{N}, N=2,3$, be a bounded open set such that

- $\partial D$ is Lipschitz,

- there exists $\rho \in(0,1 / 2]$ such that ${ }^{3} B_{\rho h} \subseteq D, h:=\operatorname{diam} D$,

- there exists $0<\rho_{0} \leq \rho$ such that $D$ is star-shaped with respect to $B_{\rho_{0} h}$.

These assumptions, stronger than those of [18], are needed in order to prove approximation results.

Definition 2.2. Given a positive number $\omega$, we define the Vekua operator $V_{1}$ and the inverse Vekua operator $V_{2}$ for the Helmholtz equation:

$$
\begin{gathered}
V_{1}, V_{2}: C(D) \rightarrow C(D), \\
V_{j}[\phi](\boldsymbol{x})=\phi(\boldsymbol{x})+\int_{0}^{1} M_{j}(\boldsymbol{x}, t) \phi(t \boldsymbol{x}) \mathrm{d} t \quad \forall \phi \in C(D), \forall \boldsymbol{x} \in D, j=1,2,
\end{gathered}
$$

where $C(D)$ is the space of the complex-valued continuous functions on $D$. The two continuous functions

$$
M_{1}, M_{2}: D \times[0,1) \rightarrow \mathbb{R}
$$

are defined as

$$
\begin{aligned}
& M_{1}(\boldsymbol{x}, t)=-\frac{\omega|\boldsymbol{x}|}{2} \frac{\sqrt{t}^{N-2}}{\sqrt{1-t}} J_{1}(\omega|\boldsymbol{x}| \sqrt{1-t}), \\
& M_{2}(\boldsymbol{x}, t)=-\frac{i \omega|\boldsymbol{x}|}{2} \frac{\sqrt{t}^{N-3}}{\sqrt{1-t}} J_{1}(i \omega|\boldsymbol{x}| \sqrt{t(1-t)}),
\end{aligned}
$$

and $J_{1}$ denotes the 1st-order Bessel function of the first kind.

Theorem 2.5 of [18] proves that these operators map harmonic functions into solutions of the Helmholtz equation and vice versa.

Theorem 2.3. Let $D$ be a domain as in Assumption 2.1; the Vekua operators satisfy:

(i) $V_{2}$ is the inverse of $V_{1}$ :

$$
V_{1}\left[V_{2}[\phi]\right]=V_{2}\left[V_{1}[\phi]\right]=\phi \quad \forall \phi \in C(D) .
$$

(ii) If $\phi$ is harmonic in $D$, i.e., $\Delta \phi=0$ in $D$, then

$$
\Delta V_{1}[\phi]+\omega^{2} V_{1}[\phi]=0 \text { in } D ;
$$

if $u$ is a solution of the homogeneous Helmholtz equation with wavenumber $\omega>0$ in $D$, i.e., $\Delta u+$ $\omega^{2} u=0$ in $D$, then

$$
\Delta V_{2}[u]=0 \quad \text { in } D .
$$

We summarize the continuity properties of the operators $V_{1}$ and $V_{2}$ that we will use in the following. For the proofs, we refer to Theorem 1.2.1 of [12] or Theorem 3.1 of [18].

\footnotetext{
${ }^{3}$ For balls, we write $B_{r}\left(\boldsymbol{x}_{\mathbf{0}}\right):=\left\{\boldsymbol{x} \in \mathbb{R}^{N},\left|\boldsymbol{x}-\boldsymbol{x}_{\mathbf{0}}\right|<r\right\}, B_{r}:=B_{r}(\mathbf{0})$.
} 
Theorem 2.4. Let $D$ be a domain as in the Assumption 2.1; the Vekua operators satisfy the following continuity bounds:

$$
\begin{aligned}
\left\|V_{1}[\phi]\right\|_{j, \omega, D} \leq & C_{N} \rho^{\frac{1-N}{2}}(1+j)^{\frac{3}{2} N+\frac{1}{2}} \mathrm{e}^{j}\left(1+(\omega h)^{2}\right)\|\phi\|_{j, \omega, D} \\
& \forall \phi \in H^{j}(D), \quad \Delta \phi=0, j \geq 0 ; \\
\left\|V_{2}[u]\right\|_{0, D} \leq & C_{N} \rho^{\frac{1-N}{2}}\left(1+(\omega h)^{4}\right) \mathrm{e}^{\frac{1}{2}(1-\rho) \omega h}\left(\|u\|_{0, D}+h|u|_{1, D}\right) \\
& \forall u \in H^{1}(D), \quad \Delta u+\omega^{2} u=0 ; \\
\left\|V_{2}[u]\right\|_{j, \omega, D} \leq & C_{N} \rho^{\frac{1-N}{2}}(1+j)^{2 N-1} e^{j}\left(1+(\omega h)^{4}\right) \mathrm{e}^{\frac{3}{4}(1-\rho) \omega h}\|u\|_{j, \omega, D} \\
& \forall u \in H^{j}(D), \quad \Delta u+\omega^{2} u=0, j \geq 1 ; \\
\left\|V_{2}[u]\right\|_{L^{\infty}(D)} \leq & \left(1+\frac{((1-\rho) \omega h)^{2}}{4} \mathrm{e}^{\left.\frac{1}{2}(1-\rho) \omega h\right)\|u\|_{L^{\infty}(D)}}\right. \\
& \forall u \in L^{\infty}(D), \quad \Delta u+\omega^{2} u=0,
\end{aligned}
$$

where the constant $C_{N}$ depends only on the space dimension $N=2,3$.

These operators and their continuity properties can be generalized to complex $\omega$, i.e., Helmholtz equation in lossy materials, see [12, Remarks 1.1.6 and 1.2.6].

We use the Vekua operators to define a class of functions that will act as intermediate elements in our approximation theory: they will approximate the general solutions of the Helmholtz equation (Sect. 3) and, in turn, will be approximated by plane waves (Sect. 4).

Definition 2.5. A function $u \in C(D)$ is called a generalized harmonic polynomial of degree $L$ if its inverse Vekua transform $V_{2}[u]$ is a harmonic polynomial of degree $L$.

In Sect. 1.3 of [12], the explicit expressions of the generalized harmonic polynomial are computed. If $N=2$, identifying $\mathbb{R}^{2}=\mathbb{C}$ and using the complex variable $z=r \mathrm{e}^{i \psi}$, we have

$$
P(z)=\sum_{l=-L}^{L} a_{l} r^{|l|} \mathrm{e}^{i l \psi} \Rightarrow V_{1}[P](z)=\sum_{l=-L}^{L} a_{l}|l| !\left(\frac{2}{\omega}\right)^{|l|} \mathrm{e}^{i l \psi} J_{|l|}(\omega r) .
$$

If $N=3$, using the spherical Bessel function $j_{\nu}(z)=\sqrt{\frac{\pi}{2 z}} J_{\nu+\frac{1}{2}}(z)$, we have

$$
\begin{aligned}
P(\boldsymbol{x}) & =\sum_{l=0}^{L} \sum_{m=-l}^{l} a_{l, m}|\boldsymbol{x}|^{l} Y_{l, m}\left(\frac{\boldsymbol{x}}{|\boldsymbol{x}|}\right) \\
& \Rightarrow V_{1}[P](\boldsymbol{x})=\sum_{l=0}^{L} \sum_{m=-l}^{l} a_{l, m} \frac{(2 l+1) !}{l !}\left(\frac{1}{2 \omega}\right)^{l} Y_{l, m}\left(\frac{\boldsymbol{x}}{|\boldsymbol{x}|}\right) j_{l}(\omega|\boldsymbol{x}|),
\end{aligned}
$$

where $\left\{Y_{l, m}\right\}_{m=-l, \ldots, l}$ are a basis of spherical harmonics of order $l$ (see $[6,14,19]$ or the Appendix of [12]). This means that the generalized harmonic polynomials in $2 \mathrm{D}$ and $3 \mathrm{D}$ are the well-known circular and spherical waves, respectively.

\section{Approximation of Helmholtz solutions by generalized harmonic polynomials}

Vekua's theory can be used to transfer the approximation properties of harmonic functions by harmonic polynomials to Helmholtz solutions by generalized harmonic polynomials.

In order to write explicitly the orders of convergence for two-dimensional domains, we introduce the following definition. 
Definition 3.1. We say that the domain $D \subset \mathbb{R}^{2}=\mathbb{C}$ satisfies the exterior cone condition with angle $\lambda \pi, \lambda \in(0,1]$ if for every $z \in \mathbb{C} \backslash D$ there is a cone $C \subset \mathbb{C} \backslash D$ with vertex in $z$ and congruent to

$$
C_{0}(\lambda \pi, r)=\{w \in \mathbb{C}|0<\arg w<\lambda \pi,| w \mid<r\} .
$$

It can be seen that if a domain $D$ satisfies Assumption 2.1, then it satisfies also the exterior cone condition with parameter $\lambda \geq \frac{2}{\pi} \arcsin \left(\frac{\rho_{0}}{1-\rho}\right)$. Any convex domain satisfies the exterior cone condition with angle $\pi(\lambda=1)$, while for a general smooth $\left(C^{1}\right)$ domain $\lambda=1-\epsilon$ is required.

Vekua's theory allows to reduce the problem of the approximation of Helmholtz solutions by generalized harmonic polynomials to the simpler case of the approximation of harmonic functions by harmonic polynomials. Concerning this problem, Theorem 2.9 of [16] provides convergence both in $h$ and $p$ in Sobolev norms for two-dimensional domains. The proof of this result is strongly based on complex analysis techniques, so it can not be directly extended to higher dimensions. In Chapter 2 of [12], we have generalized that estimate to higher space dimensions. We summarize these results in the following theorem.

Theorem 3.2. Let $D$ be a domain as in Assumption 2.1, $k \in \mathbb{N}$ and $u \in H^{k+1}(D)$ be a solution of the homogeneous Helmholtz equation $\Delta u+\omega^{2} u=0$ in $D$. Then, the following results hold.

(i) If $N=2$ and $D$ satisfies the exterior cone condition with angle $\lambda \pi$, then for every $L \geq k$, there exists a generalized harmonic polynomial $Q_{L}^{\prime}$ of degree at most $L$ such that, for every $j \leq k+1$, it holds

$$
\left\|u-Q_{L}^{\prime}\right\|_{j, \omega, D} \leq C\left(1+(\omega h)^{j+6}\right) \mathrm{e}^{\frac{3}{4}(1-\rho) \omega h}\left(\frac{\log (L+2)}{L+2}\right)^{\lambda(k+1-j)} h^{k+1-j}\|u\|_{k+1, \omega, D},
$$

where the constant $C$ depends only on the shape of $D, j$ and $k$, but is independent of $h, \omega, L$ and $u$.

(ii) If $N=3$, there exists a constant $\lambda>0$ depending only on the shape of $D$, such that for every $L \geq \max \left\{k, 2^{1 / \lambda}\right\}$, there exists a generalized harmonic polynomial $Q_{L}^{\prime \prime}$ of degree at most $L$ such that, for every $j \leq k+1$, it holds

$$
\left\|u-Q_{L}^{\prime \prime}\right\|_{j, \omega, D} \leq C\left(1+(\omega h)^{j+6}\right) \mathrm{e}^{\frac{3}{4}(1-\rho) \omega h} L^{-\lambda(k+1-j)} h^{k+1-j}\|u\|_{k+1, \omega, D},
$$

where the constant $C$ depends only on the shape of $D, j$ and $k$, but is independent of $h, \omega, L$ and $u$.

Part (i) of Theorem 3.2 is a simple consequence of (4), Theorem 2.9 of [16] and (6); the proof of part (ii) is given in the Appendix.

Theorem 3.2 shows that a solution of the Helmholtz equation with Sobolev regularity $k+1$ can be approximated by generalized harmonic polynomials with algebraic convergence both in the mesh size $h$ and in the degree $L$. The order of convergence in $h$ is $k+1-j$, and the order of convergence in $L$ is $\lambda(k+1-j)$, where $\lambda$ is a parameter depending on the domain shape.

The two-dimensional result comes from [16]; in this case, we have complete control of the speed of convergence, since $\pi \lambda$ is the opening of the smallest reentrant corner of the domain; estimate (10) has been shown in [16] to be sharp.

In three-dimensions, the result is much less powerful because an explicit lower bound to the parameter $\lambda$ in (11) is not available. This means that the convergence rate in $L$ is not fully explicit: this is the main gap in the approximation theory presented here. So far, we could not prove an explicit bound for $\lambda$ even in the simple cases where $D$ is a cube or a regular tetrahedron.

Remark 3.3. If $u$ with $\Delta u+\omega^{2} u=0$ possesses an analytic extension beyond $\partial D$, then, thanks to Theorem A.1, we can expect exponentially accurate approximation by generalized harmonic polynomials, in the sense that

$$
\exists \gamma=\gamma(u, D, j, \omega)>0: \inf _{Q \in H P_{\omega, L}}\|u-Q\|_{j, \omega, D} \leq C(u, D, j, \omega) \exp (-\gamma L) \quad \forall L \in \mathbb{N},
$$


see [16, Corollary 2.7]. Below we will show that also the second term in (3) converges exponentially in $p$ (see Lemmas 4.3 and 4.7), so that overall exponential convergence of plane wave approximation is guaranteed.

\section{Approximation of generalized harmonic polynomials by plane waves}

Now, we want to approximate the generalized harmonic polynomials using linear combinations of plane waves. The link between plane and circular/spherical waves is given by the Jacobi-Anger expansion, combined with the addition theorem for spherical harmonics (see (2.29), (2.45) and (3.66) in [6]):

$$
\begin{aligned}
2 D: & \mathrm{e}^{i r \cos \theta}=\sum_{l \in \mathbb{Z}} i^{l} J_{l}(r) \mathrm{e}^{i l \theta} \quad \forall r \geq 0, \theta \in[0,2 \pi], \\
3 D: & \mathrm{e}^{i r \boldsymbol{\xi} \cdot \boldsymbol{\eta}}=4 \pi \sum_{l \geq 0} i^{l} j_{l}(r) \sum_{m=-l}^{l} Y_{l, m}(\boldsymbol{\xi}) \overline{Y_{l, m}(\boldsymbol{\eta})} \quad \forall r \geq 0, \boldsymbol{\xi}, \boldsymbol{\eta} \in \mathbb{S}^{2} .
\end{aligned}
$$

In what follows, we will always consider plane wave spaces with dimension $p$ chosen according to

$$
p=\operatorname{dim} H P_{\omega, q}\left(\mathbb{R}^{N}\right)= \begin{cases}2 q+1 & \text { in two-dimensions } \\ (q+1)^{2} & \text { in three-dimensions }\end{cases}
$$

for some $q \in \mathbb{N}$.

We pursue the following policy: given a generalized harmonic polynomial to be approximated, we represent it as a (finite) linear combination of circular/spherical waves (see (8) and (9)); then, we truncate the Jacobi-Anger expansion of the generic element $\sum_{k=1}^{p} \alpha_{k} \mathrm{e}^{i \omega \boldsymbol{x} \cdot \boldsymbol{d}_{k}}$ of $P W_{\omega, p}\left(\mathbb{R}^{N}\right)$, "solve" the resulting linear system with the $\alpha_{k}$ 's as unknowns and thus define the approximating function in $P W_{\omega, p}\left(\mathbb{R}^{N}\right)$. Error bounds will be obtained by estimating the residual error produced by the truncation of the JacobiAnger expansions. We will do this in Lemma 4.3 (two-dimensions) and Lemma 4.7 (three-dimensions): this entails bounding the norm of the inverse of a matrix defined by the generalized harmonic polynomials. The proof will be fairly technical, because we need a very precise estimate of all the terms involved; on the other hand, we obtain a sharp algebraic order of convergence in $h$, the diameter of the domain, and a faster than exponential speed of convergence in $p$, the number of plane waves used. In the two-dimensional case, this result holds for any choice of the plane wave directions, while in three-dimensions, we will have to choose them carefully.

\subsection{Tool: stable bases}

Our analysis relies on the existence of a basis of the plane wave space that does not degenerate for small wavenumbers. Yet, it is well-known that the plane wave Galerkin matrix associated with the $L^{2}(D)$ inner product (mass matrix) is very ill-conditioned when the wave number is small or when the size of the domain is small, because in these cases the plane waves tend to be linearly dependent. In order to cope with this problem, it is possible to introduce a basis for the space $P W_{\omega, p}\left(\mathbb{R}^{N}\right)$ that is stable with respect to this limit.

In $2 \mathrm{D}$, a stable basis was introduced in [11, Sect. 3.1]. Here, we give a simpler construction:

$$
b_{l}(\boldsymbol{x}):=(-i)^{l} \gamma_{l}|l| !\left(\frac{2}{\omega}\right)^{|l|} \sum_{l^{\prime}=-q}^{q}\left(\boldsymbol{A}^{-t}\right)_{l ; l^{\prime}} \mathrm{e}^{i \omega \boldsymbol{x} \cdot \boldsymbol{d}_{l^{\prime}}} \quad l=-q, \ldots, q,
$$

where $\gamma_{l}=1$ if $l \geq 0$ and $\gamma_{l}=(-1)^{l}$ if $l<0$. The plane waves directions are

$$
\boldsymbol{d}_{l}=\left(\cos \theta_{l}, \sin \theta_{l}\right) \quad l=-q, \ldots, q, \quad \boldsymbol{d}_{l} \neq \boldsymbol{d}_{k} \forall l \neq k,
$$

and the matrix $\boldsymbol{A}$ is

$$
\boldsymbol{A}=\left\{\boldsymbol{A}_{l ; l^{\prime}}\right\}_{\substack{l=-q, \ldots, q \\ l^{\prime}=-q, \ldots, q}}=\left\{\mathrm{e}^{-i l \theta_{l^{\prime}}}\right\}_{\substack{l=-q, \ldots, q \\ l^{\prime}=-q, \ldots, q}} \in \mathbb{C}^{2 q+1,2 q+1} .
$$


With this definition, using the polar coordinates $\boldsymbol{x}=r(\cos \psi, \sin \psi)$, we have

$$
\begin{aligned}
b_{l}(\boldsymbol{x}) & =(-i)^{l} \gamma_{l}|l| !\left(\frac{2}{\omega}\right)^{|l|} \sum_{l^{\prime}=-q}^{q}\left(\boldsymbol{A}^{-t}\right)_{l ; l^{\prime}} \mathrm{e}^{i \omega r \cos \left(\psi-\theta_{l^{\prime}}\right)} \\
& \stackrel{(13)}{=}(-i)^{l} \gamma_{l}|l| !\left(\frac{2}{\omega}\right)^{|l|} \sum_{\tilde{l} \in \mathbb{Z}} i^{\tilde{l}} J_{\tilde{l}}(\omega r) \mathrm{e}^{i \tilde{l} \psi} \sum_{l^{\prime}=-q}^{q}\left(\boldsymbol{A}^{-t}\right)_{l ; l^{\prime}} \mathrm{e}^{-i \tilde{\theta} \theta_{l^{\prime}}} \\
& =(-i)^{l} \gamma_{l}|l| !\left(\frac{2}{\omega}\right)^{|l|}\left(i^{l} J_{l}(\omega r) \mathrm{e}^{i l \psi}+\sum_{|\tilde{l}|>q} i^{\tilde{l}} J_{\tilde{l}}(\omega r) \mathrm{e}^{i \tilde{l} \psi} \sum_{l^{\prime}=-q}^{q}\left(\boldsymbol{A}^{-t}\right)_{l ; l^{\prime}} \mathrm{e}^{-i \tilde{l} \theta_{l^{\prime}}}\right) \\
& \stackrel{(8)}{=} V_{1}\left[r^{|l|} \mathrm{e}^{i l \psi}\right]+O\left(\omega^{q+1-|l|}\right)_{\omega \rightarrow 0},
\end{aligned}
$$

where we used the property $J_{-k}(z)=(-1)^{k} J_{k}(z) \forall k \in \mathbb{Z}$.

In three-dimensions, thanks to the Jacobi-Anger expansion and the definition of the generalized harmonic polynomials, we can easily find a stable basis for $P W_{\omega, p}\left(\mathbb{R}^{3}\right)$.

We fix $q \in \mathbb{N}, p=(q+1)^{2}$ and the $p$ directions $\left\{\boldsymbol{d}_{l, m}\right\}_{l=0, \ldots, q ;|m| \leq l}$, which define $P W_{\omega, p}\left(\mathbb{R}^{3}\right)$ in such a way that the $p \times p$ matrix ${ }^{4}$

$$
\boldsymbol{M}=\left\{\boldsymbol{M}_{l, m ; l^{\prime}, m^{\prime}}\right\}_{\substack{l=0, \ldots, q,|m| \leq l, l^{\prime}=0, \ldots, q,\left|m^{\prime}\right| \leq l^{\prime}}}=\left\{Y_{l, m}\left(\boldsymbol{d}_{l^{\prime}, m^{\prime}}\right)\right\}_{\substack{l=0, \ldots, q,|m| \leq l \\ l^{\prime}=0, \ldots, q,\left|m^{\prime}\right| \leq l^{\prime}}}
$$

is invertible. We define $p$ elements of $P W_{\omega, p}\left(\mathbb{R}^{3}\right)$

$$
\begin{gathered}
b_{l, m}(\boldsymbol{x})=\frac{\Gamma\left(l+\frac{3}{2}\right)}{2 \pi^{\frac{3}{2}}}\left(\frac{2}{i \omega}\right)^{l} \sum_{\substack{l^{\prime}=0, \ldots, q,\left|m^{\prime}\right| \leq l^{\prime}}}\left(\overline{\boldsymbol{M}^{-t}}\right)_{l, m ; l^{\prime}, m^{\prime}} \mathrm{e}^{i \omega \boldsymbol{x} \cdot \boldsymbol{d}_{l^{\prime}, m^{\prime}}} \\
l=0, \ldots, q, \quad|m| \leq l .
\end{gathered}
$$

Relying on the Jacobi-Anger expansion (14), we obtain:

$$
\begin{aligned}
& b_{l, m}(\boldsymbol{x})= 4 \pi \frac{\Gamma\left(l+\frac{3}{2}\right)}{2 \pi^{\frac{3}{2}}}\left(\frac{2}{i \omega}\right)^{l} \sum_{\substack{\tilde{l} \in \mathbb{N},|\tilde{m}| \leq \tilde{l}}} i^{\tilde{l}} j_{\tilde{l}}(\omega|\boldsymbol{x}|) Y_{\tilde{l}, \tilde{m}}\left(\frac{\boldsymbol{x}}{|\boldsymbol{x}|}\right) \sum_{\substack{l^{\prime}=0, \ldots, q,\left|m^{\prime}\right| \leq l^{\prime}}}\left(\overline{\boldsymbol{M}^{-1}}\right)_{l^{\prime}, m^{\prime} ; l, m} \overline{Y_{\tilde{l}, \tilde{m}}\left(\boldsymbol{d}_{l^{\prime}, m^{\prime}}\right)} \\
&= \frac{2 \Gamma\left(l+\frac{3}{2}\right)}{\sqrt{\pi}}\left(\frac{2}{i \omega}\right)^{l}\left[i^{l} j_{l}(\omega|\boldsymbol{x}|) Y_{l, m}\left(\frac{\boldsymbol{x}}{|\boldsymbol{x}|}\right)\right. \\
&\left.+\sum_{\substack{\tilde{l}>q,|\tilde{m}| \leq \tilde{l}}} i^{\tilde{l}} j_{\tilde{l}}(\omega|\boldsymbol{x}|) Y_{\tilde{l}, \tilde{m}}\left(\frac{\boldsymbol{x}}{|\boldsymbol{x}|}\right)_{\substack{l^{\prime}=0, \ldots, q,\left|m^{\prime}\right| \leq l^{\prime}}}\left(\overline{\boldsymbol{M}^{-1}}\right)_{l^{\prime}, m^{\prime} ; l, m} \overline{Y_{\tilde{l}, \tilde{m}}\left(\boldsymbol{d}_{l^{\prime}, m^{\prime}}\right)}\right] \\
& \stackrel{(9)}{=} V_{1}\left[|\boldsymbol{x}|^{l} Y_{l, m}\left(\frac{\boldsymbol{x}}{|\boldsymbol{x}|}\right)\right]+O\left(\omega^{q+1-l}\right)_{\omega \rightarrow 0},
\end{aligned}
$$

${ }^{4}$ Since vector indices are often denoted by a pair of integers separated by a comma (e.g., $\left.\boldsymbol{d}_{l, m}\right)$, here and in the following, we use the semicolon to separate the row and column indices of second-order matrices (e.g., $\boldsymbol{M}_{l, m ; l^{\prime}, m^{\prime}}$ ). The components of vectors and matrices will be denoted by round brackets with subscripts, whenever their names are composite (e.g., $(\boldsymbol{M} \boldsymbol{d})_{l, m}$ or $\left.\left(\overline{\boldsymbol{M}^{-1}}\right)_{l, m ; l^{\prime}, m^{\prime}}\right)$. The superscript ${ }^{-t}$ will be used to denote the transpose of the inverse (i.e., $\left.\boldsymbol{M}^{-t}=\left(\boldsymbol{M}^{-1}\right)^{t}\right)$. 
thanks to the asymptotic properties of the spherical Bessel functions for small arguments

$$
j_{k}(z) \approx \frac{2^{k} k !}{(2 k+1) !} z^{k} \quad|z|<<1, k \in \mathbb{Z}
$$

and to

$$
\begin{aligned}
\sum_{\substack{l^{\prime}=0, \ldots, q,\left|m^{\prime}\right| \leq l^{\prime}}}\left(\boldsymbol{M}^{-1}\right)_{l^{\prime}, m^{\prime} ; l, m} Y_{\tilde{l}, \tilde{m}}\left(\boldsymbol{d}_{l^{\prime}, m^{\prime}}\right) & =\sum_{\substack{l^{\prime}=0, \ldots, q,\left|m^{\prime}\right| \leq l^{\prime}}}\left(\boldsymbol{M}^{-1}\right)_{l^{\prime}, m^{\prime} ; l, m}(\boldsymbol{M})_{\tilde{l}, \tilde{m} ; l^{\prime}, m^{\prime}} \\
& =\delta_{l, \tilde{l}} \delta_{m, \tilde{m},}, \quad \text { if }|\tilde{m}| \leq \tilde{l} \leq q .
\end{aligned}
$$

The functions $b_{l, m}$ constitute a basis in $P W_{\omega, p}\left(\mathbb{R}^{3}\right)$; since

$$
b_{l, m}(\boldsymbol{x}) \stackrel{\omega \rightarrow 0}{\longrightarrow}|\boldsymbol{x}|^{l} Y_{l, m}\left(\frac{\boldsymbol{x}}{|\boldsymbol{x}|}\right)
$$

uniformly on compact sets, this basis does not degenerate for small positive $\omega$ and its associated mass matrix is well conditioned.

The existence of a stable basis and the proof of the convergence of the plane wave approximation require the matrices $\boldsymbol{A}$ and $\boldsymbol{M}$ to be invertible. This is the case if and only if the sets of directions $\left\{\boldsymbol{d}_{l}\right\}$ or $\left\{\boldsymbol{d}_{l, m}\right\}$ (in two- or three-dimensions, respectively) constitute a fundamental system for the harmonic polynomials of degree at most $q$. In two-dimensions, if the directions $\boldsymbol{d}_{l}$ are all different from each other, this is always true, as we will see in the proof of Lemma 4.3. In three-dimensions, we prove that there exist many configurations of directions that make $\boldsymbol{M}$ invertible in the following two lemmas and provide an example.

Lemma 4.1. Let the matrix $\boldsymbol{M}$ be defined as in (16). The set of the configurations of directions $\left\{\boldsymbol{d}_{l, m}\right\}_{l=0, \ldots, q,|m| \leq l}$ that makes $\boldsymbol{M}$ invertible is a dense open subset of $\left(\mathbb{S}^{2}\right)^{p}$.

Proof. The spherical harmonics $Y_{l, m}=Y_{l, m}(\sin \theta \cos \varphi, \sin \theta \sin \varphi, \cos \theta)$, and thus the determinant $\operatorname{det}(\boldsymbol{M}):\left(\mathbb{S}^{2}\right)^{p} \rightarrow \mathbb{C}$, are polynomial functions of $\sin \theta, \cos \theta, \sin \varphi, \cos \varphi$. This implies that $\operatorname{det}(\boldsymbol{M})$ is continuous, and then, its pre-image $[\operatorname{det}(\boldsymbol{M})]^{-1}\{\mathbb{C} \backslash 0\}$ is an open set.

The existence of at least one configuration of directions $\left\{\boldsymbol{d}_{l, m}\right\}_{l=0, \ldots, q ;|m| \leq l}$ such that $\boldsymbol{M}$ is invertible is guaranteed by a simple generalization (to non-constant degrees $n$ ) of Lemma 6 of [19] or by Lemma 4.2 below. Since a trigonometric polynomial is equal to zero in an open set of $\mathbb{R}^{2 p}$ if and only if it is zero everywhere, then $\operatorname{det}(\boldsymbol{M})$ is zero only in a closed subset of $\left(\mathbb{S}^{2}\right)^{p}$ with empty interior, which means that $M$ is invertible on a dense set.

Lemma 4.2. Given $q \in \mathbb{N}$, let the $p=(q+1)^{2}$ directions on $\mathbb{S}^{2}$ be chosen as

$$
\boldsymbol{d}_{l, m}=\left(\sin \theta_{l} \cos \varphi_{l, m}, \sin \theta_{l} \sin \varphi_{l, m}, \cos \theta_{l}\right)
$$

for all $l=0, \ldots, q,|m| \leq l$, where the $q+1$ colatitude angles $\left\{\theta_{l}\right\}_{l=0, \ldots, q} \subset(0, \pi)$ are all different from each other, and the azimuths $\left\{\varphi_{l, m}\right\}_{l=0, \ldots, q ;|m| \leq l} \subset[0,2 \pi)$ satisfy $\varphi_{l, m} \neq \varphi_{l, m^{\prime}}$ for every $m \neq m^{\prime}$. Then, the matrix $\boldsymbol{M}$ defined in (16) is invertible.

Proof. The proof is quite technical, and we refer the interested reader to [12] (see Lemma 3.1.2).

Lemma 4.2 provides a quite general class of configurations of plane wave propagation directions $\left\{\boldsymbol{d}_{l, m}\right\}_{l=0, \ldots, q ;|m| \leq l}$ that renders the matrix $\boldsymbol{M}$ invertible. This implies the existence of a stable basis in $P W_{\omega, p}\left(\mathbb{R}^{3}\right)$ and allows to prove the approximation estimates in $h$ in Sect. 4.3. To prove estimates in $p$, we will need a smarter choice of the directions. In order to fulfill the hypotheses of Lemma 4.2, the directions only have to satisfy the following geometric requirement: there exists $q+1$ different heights $z_{j} \in(-1,1)$ such that exactly $2 j+1$ different vectors $\boldsymbol{d}_{l, m}$ belong to $\mathbb{S}^{2} \cap\{(x, y, z)$, $\left.z=z_{j}\right\}_{j=0, \ldots, q}$.

An example of directions satisfying this condition with $q=3$ is shown in Fig. 1. 


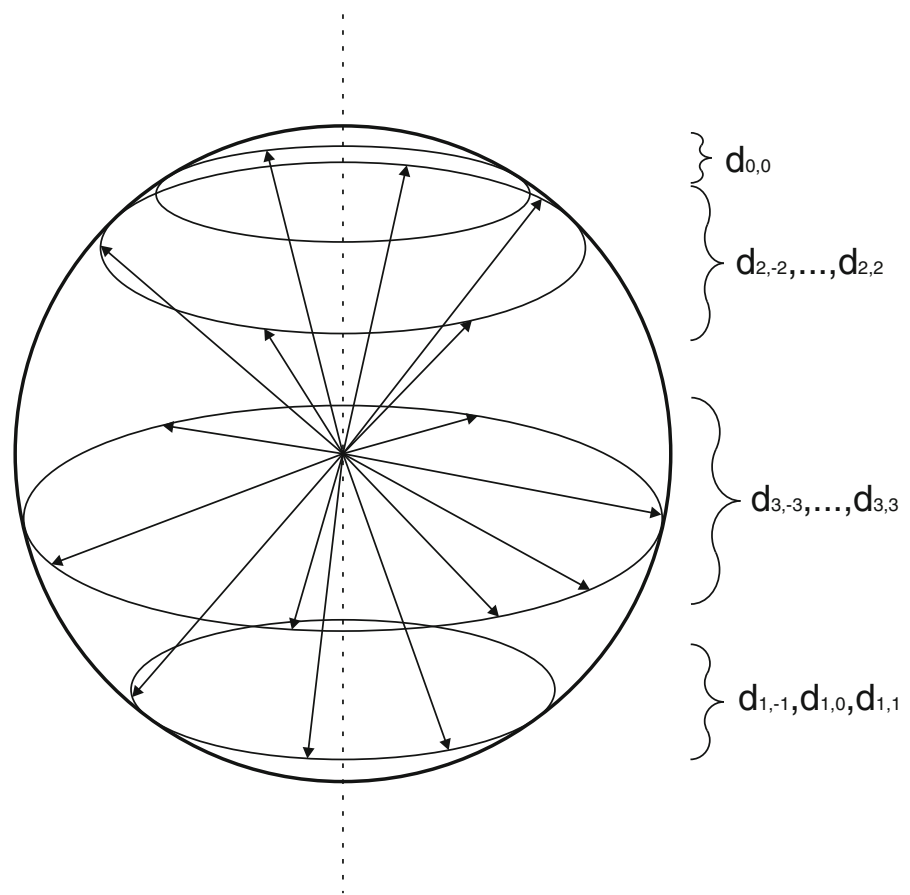

FIG. 1. A choice of directions $\left\{\boldsymbol{d}_{l, m}\right\}_{l=0, \ldots, q ;|m| \leq l}$ that satisfies the hypothesis of Lemma 4.2 with $q=3, p=16$. Notice that 1 direction belongs to level 0,3 directions to level 1,5 to level 2 and 7 to level 3

\subsection{The two-dimensional case}

In two space dimensions, thanks to the Jacobi-Anger expansion and the special properties of the circular harmonics $Y_{l}(\theta)=\mathrm{e}^{i l \theta}$, we can approximate a generalized harmonic polynomial in $P W_{\omega, p}\left(\mathbb{R}^{2}\right)$, with completely explicit error estimates both in $h$ and in $p$. The order of convergence with respect to $h$ is sharp, as it can be seen from simple numerical experiments $[4,10,11,17]$. The proof given below improves considerably the one given in [17].

Lemma 4.3. Let $D \subset \mathbb{R}^{2}$ be a domain as in Assumption 2.1. Let $P$ be a harmonic polynomial of degree $L$ and let

$$
\left\{\boldsymbol{d}_{k}=\left(\cos \theta_{k}, \sin \theta_{k}\right)\right\}_{k=-q, \ldots, q}
$$

be the different directions in the definition of $P W_{\omega, p}\left(\mathbb{R}^{2}\right), p=2 q+1$. We assume that there exists $0<\delta \leq 1$ such that

$$
\min _{\substack{j, k=-q, \ldots, q \\ j \neq k}}\left|\theta_{j}-\theta_{k}\right| \geq \frac{2 \pi}{p} \delta
$$

Let the conditions on the indices

$$
0 \leq K \leq L \leq q, \quad L-K \leq\left\lfloor\frac{q-1}{2}\right\rfloor,
$$

be satisfied. Then, there exists a vector $\vec{\alpha} \in \mathbb{C}^{p}$ such that, for every $R>0$,

$$
\left\|V_{1}[P]-\sum_{k=-q}^{q} \alpha_{k} \mathrm{e}^{i \omega \boldsymbol{x} \cdot \boldsymbol{d}_{k}}\right\|_{L^{\infty}\left(B_{R}\right)} \leq C(\omega, \delta, \rho, h, R, q, K, L)\|P\|_{K, \omega, D},
$$


where we have set, for brevity,

$$
\begin{aligned}
C(\omega, \delta, \rho, h, R, q, K, L)= & \frac{\mathrm{e}^{3}}{\pi^{\frac{3}{2}} \rho^{L-K+1}}\left(\frac{\mathrm{e}^{\frac{5}{2}}}{2 \sqrt{2} \delta^{2}}\right)^{q}\left(2^{L} \sqrt{L+1}\right) \\
& \cdot(\omega R)^{q+1-K}\left(1+(\omega h)^{-L+K}\right) \mathrm{e}^{\frac{\omega R}{2}} \frac{R^{K}}{h} \frac{1}{(q+1)^{\frac{q+1}{2}}} .
\end{aligned}
$$

Proof. We write the polynomial

$$
P(z)=\sum_{l=-L}^{L} a_{l} r^{|l|} \mathrm{e}^{i l \psi}
$$

with the usual identification $\mathbb{R}^{2}=\mathbb{C}$ and $z=r \mathrm{e}^{i \psi}$. We have

$$
\begin{aligned}
& V_{1}[P](z)-\sum_{k=-q}^{q} \alpha_{k} \mathrm{e}^{i \omega(r \cos \psi, r \sin \psi) \cdot \boldsymbol{d}_{k}} \\
& \stackrel{(8)}{=} \sum_{l=-L}^{L} a_{l}|l| !\left(\frac{2}{\omega}\right)^{|l|} \mathrm{e}^{i l \psi} J_{|l|}(\omega r)-\sum_{k=-q}^{q} \alpha_{k} \mathrm{e}^{i \omega r \cos \left(\psi-\theta_{k}\right)} \\
& \stackrel{(13)}{=} \sum_{l=-L}^{L} a_{l}|l| !\left(\frac{2}{\omega}\right)^{|l|} \mathrm{e}^{i l \psi} \gamma_{l} J_{l}(\omega r)-\sum_{l \in \mathbb{Z}} i^{l} J_{l}(\omega r) \mathrm{e}^{i l \psi} \sum_{k=-q}^{q} \alpha_{k} \mathrm{e}^{-i l \theta_{k}}
\end{aligned}
$$

where $\gamma_{l}=1$ if $l \geq 0$ and $\gamma_{l}=(-1)^{l}$ if $l<0$ because $J_{-l}(\omega r)=(-1)^{l} J_{l}(\omega r)$. Define the $p \times p$ matrix $\boldsymbol{A}$ by

$$
\boldsymbol{A}=\left\{\boldsymbol{A}_{l ; k}\right\}_{l, k=-q, \ldots, q}=\left\{\mathrm{e}^{-i l \theta_{k}}\right\}_{l, k=-q, \ldots, q},
$$

and the vector $\vec{\beta} \in \mathbb{C}^{p}$ by

$$
\beta_{l}= \begin{cases}a_{l}|l| !\left(\frac{2}{\omega}\right)^{|l|} i^{-l} \gamma_{l}, & l=-L, \ldots, L \\ 0, & l=-q, \ldots,-L-1, L+1, \ldots, q .\end{cases}
$$

The matrix $\boldsymbol{A}$ is non-singular because it is the product of a Vandermonde matrix and a diagonal matrix:

$$
\boldsymbol{A}=\left\{\mathrm{e}^{-i j \theta_{k}}\right\}_{\substack{j=0, \ldots, 2 q \\ k=-q, \ldots, q}} \cdot \operatorname{diag}\left(\left\{\mathrm{e}^{i q \theta_{k}}\right\}_{k=-q, \ldots, q}\right)=\boldsymbol{V}_{A} \cdot \boldsymbol{D}_{A}
$$

By choosing the $p$-dimensional vector $\vec{\alpha}$ as the solution of the linear system $\boldsymbol{A} \vec{\alpha}=\vec{\beta}$, we have

$$
V_{1}[P](z)-\sum_{k=-q}^{q} \alpha_{k} \mathrm{e}^{i \omega(r \cos \psi, r \sin \psi) \cdot \boldsymbol{d}_{k}}=-\sum_{|l|>q} i^{l} J_{l}(\omega r) \mathrm{e}^{i l \psi} \sum_{k=-q}^{q} \alpha_{k} \mathrm{e}^{-i l \theta_{k}}
$$

and thus, the $L^{\infty}$ norm of the error is controlled by

$$
\left\|V_{1}[P]-\sum_{k=-q}^{q} \alpha_{k} \mathrm{e}^{i \omega \boldsymbol{x} \cdot \boldsymbol{d}_{k}}\right\|_{L^{\infty}\left(B_{R}\right)} \leq\left(\sup _{t \in[0, \omega R]} 2 \sum_{l>q}\left|J_{l}(t)\right|\right)\left\|\boldsymbol{A}^{-1}\right\|_{1}\|\vec{\beta}\|_{1} .
$$

We have to bound each of the three factors on the right-hand side of (22).

Using the well-known bound for the Bessel functions

$$
\left|J_{\nu}(z)\right| \leq \frac{\mathrm{e}^{|\operatorname{Im} z|}}{\Gamma(\nu+1)}\left(\frac{|z|}{2}\right)^{\nu} \quad \forall \nu>-\frac{1}{2}, z \in \mathbb{C},
$$


we have, for the first factor,

$$
\begin{aligned}
\sup _{t \in[0, \omega R]} \sum_{l>q}\left|J_{l}(t)\right| & \stackrel{(23)}{\leq} \sup _{t \in[0, \omega R]} \sum_{l>q}\left(\frac{t}{2}\right)^{l} \frac{1}{l !} \\
& \leq \sup _{t \in[0, \omega R]}\left(\frac{t}{2}\right)^{q+1} \frac{1}{(q+1) !} \sum_{j \geq 0}\left(\frac{t}{2}\right)^{j} \frac{1}{j !}=\left(\frac{\omega R}{2}\right)^{q+1} \frac{\mathrm{e}^{\frac{\omega R}{2}}}{(q+1) !} .
\end{aligned}
$$

For $\left\|\boldsymbol{A}^{-1}\right\|_{1}$, we observe that the 1 norm of the inverse of the diagonal matrix $\boldsymbol{D}_{A}$ is one, while the norm of the inverse of the Vandermonde matrix $\boldsymbol{V}_{A}$ can be bounded using Theorem 1 of [8]:

$$
\left\|\boldsymbol{A}^{-1}\right\|_{1} \leq\left\|\boldsymbol{V}_{A}^{-1}\right\|_{1}\left\|\boldsymbol{D}_{A}^{-1}\right\|_{1} \leq p\left\|\boldsymbol{V}_{A}^{-1}\right\|_{\infty} \leq p \max _{\substack { k=-q, \ldots, q \\
\begin{subarray}{c}{s=-q, \ldots, q \\
s \neq k{ k = - q , \ldots , q \\
\begin{subarray} { c } { s = - q , \ldots , q \\
s \neq k } }\end{subarray}} \frac{1+\left|\mathrm{e}^{-i \theta_{s}}\right|}{\mid \mathrm{e}^{-i \theta_{s}}-\mathrm{e}^{-i \theta_{k} \mid}} .
$$

With simple geometric considerations, it is easy to see that, under the constraint (18), the product on the right-hand side is bounded by its value when

$$
\theta_{s}^{*}=\theta_{0}^{*}+\frac{2 \pi}{p} \delta s \quad s=-q, \ldots, q
$$

and the maximum is obtained for $k=0$. A simple trigonometric calculation gives

$$
\left|\mathrm{e}^{-i \theta_{s}^{*}}-\mathrm{e}^{-i \theta_{0}^{*}}\right|=\sqrt{2} \sqrt{1-\cos \left(\theta_{s}^{*}-\theta_{0}^{*}\right)} \geq \sqrt{2} \frac{\sqrt{2}}{\pi}\left|\theta_{s}^{*}-\theta_{0}^{*}\right|=\frac{4}{p} \delta|s|,
$$

because $1-\cos t \geq \frac{2}{\pi^{2}} t^{2}$ for every $t \in[-\pi, \pi]$. This leads to the bound

$$
\left\|\boldsymbol{A}^{-1}\right\|_{1} \leq p \prod_{\substack{s=-q, \ldots, q \\ s \neq k}} \frac{2 p}{4 \delta|s|} \leq \frac{p^{p}}{(2 \delta)^{2 q}(q !)^{2}}
$$

In order to bound $\|\vec{\beta}\|_{1}$, we need to bound from below the Sobolev seminorm of order $\mu$ of $P$ for every $\mu=0, \ldots, L$. Recalling that $B_{\rho h} \subseteq D$ and taking into account the expression of $P$ in (21), we have

$$
\begin{aligned}
|P|_{\mu, D}^{2} & \geq\left\|\frac{\partial^{\mu}}{\partial r^{\mu}} P\right\|_{0, B_{\rho h}}^{2}=\left\|\sum_{|j|=\mu}^{L} a_{j} \frac{|j| !}{(|j|-K) !} r^{|j|-K} \mathrm{e}^{i j \psi}\right\|_{0, B_{\rho h}}^{2} \\
& =\int_{0}^{\rho h} \sum_{|j|,\left|j^{\prime}\right|=\mu}^{L} \frac{a_{j} \overline{a_{j^{\prime}}}|j| !\left|j^{\prime}\right| !}{(|j|-\mu) !\left(\left|j^{\prime}\right|-\mu\right) !} r^{|j|+\left|j^{\prime}\right|-2 \mu} \int_{0}^{2 \pi} \mathrm{e}^{i\left(j-j^{\prime}\right) \psi} \mathrm{d} \psi r \mathrm{~d} r \\
& =2 \pi \sum_{|j|=\mu}^{L}\left|a_{j}\right|^{2} \frac{(|j| !)^{2}}{((|j|-\mu) !)^{2}} \frac{(\rho h)^{2(|j|-\mu+1)}}{2(|j|-\mu+1)},
\end{aligned}
$$

where in the last step we have used the identity

$$
\int_{0}^{2 \pi} \mathrm{e}^{i\left(j-j^{\prime}\right) \psi} \mathrm{d} \psi=2 \pi \delta_{j j^{\prime}}
$$


All the terms in the sum on the right-hand side of (26) are non-negative, so we can invert the estimate. Thus, considering (26) for $\mu=|l|$ and $\mu=K$, we obtain, respectively,

$$
\begin{aligned}
& \left|a_{l}\right| \leq \frac{1}{\sqrt{\pi}} \frac{1}{|l| !(\rho h)}|P|_{|l|, D} \quad 0 \leq|l| \leq L, \\
& \left|a_{l}\right| \leq \frac{1}{\sqrt{\pi}} \frac{(|l|-K) ! \sqrt{|l|-K+1}}{|l| !(\rho h)^{|l|-K+1}}|P|_{K, D} \quad K \leq|l| \leq L .
\end{aligned}
$$

We plug these bounds into the definition of the coefficients of $\vec{\beta}$, with $K \leq L$ :

$$
\begin{aligned}
\|\vec{\beta}\|_{1} & =\sum_{l=-L}^{L}\left|a_{l}\right|\left(\frac{2}{\omega}\right)^{|l|}|l| ! \\
& \leq \sum_{l=-K}^{K} \frac{1}{\sqrt{\pi} \rho h}\left(\frac{2}{\omega}\right)^{|l|}|P|_{|l|, D}+\sum_{|l|=K+1}^{L} \frac{1}{\sqrt{\pi}}\left(\frac{2}{\omega}\right)^{|l|} \frac{(|l|-K) ! \sqrt{|l|-K+1}}{(\rho h)^{|l|-K+1}}|P|_{K, D} \\
& \leq \frac{\sqrt{2 K+1} 2^{K+\frac{1}{2}}}{\sqrt{\pi} \rho h} \omega^{-K}\|P\|_{K, \omega, D}+\frac{2^{L+1}}{\sqrt{\pi} \rho^{L-K+1} h} \omega^{-K}\left(\sum_{l=K+1}^{L} \frac{(l-K) ! \sqrt{l-K+1}}{(\omega h)^{|l|-K}}\right)|P|_{K, D} \\
& \leq\left\{\frac{2^{L+1}}{\sqrt{\pi} \rho^{L-K+1}}\left(1+(\omega h)^{-L+K}\right) \frac{\omega^{-K}}{h}(\sqrt{K+1}+(L-K)(L-K) ! \sqrt{L-K+1})\right\}\|P\|_{K, \omega, D} .
\end{aligned}
$$

Inserting the bound on the sum of the Bessel functions (24), the one on $\left\|\boldsymbol{A}^{-1}\right\|_{1}$ given by (25) and the one on $\|\vec{\beta}\|_{1}$ given by (27) inside (22) gives

$$
\begin{aligned}
\| V_{1}[P] & -\sum_{k=-q}^{q} \alpha_{k} \mathrm{e}^{i \omega \boldsymbol{x} \cdot \boldsymbol{d}_{k}}\|\|_{L^{\infty}\left(B_{R}\right)} \\
\leq & 2\left\{\left(\frac{\omega R}{2}\right)^{q+1} \frac{\mathrm{e}^{\frac{\omega R}{2}}}{(q+1) !}\right\}\left\{\frac{p^{p}}{(2 \delta)^{2 q}(q !)^{2}}\right\} \\
& \cdot\left\{\frac{2^{L+1}}{\sqrt{\pi} \rho^{L-K+1}} \omega^{-K} h^{-1}\left(1+(\omega h)^{-L+K}\right) \sqrt{L+1}(L-K+1) !\right\}\|P\|_{K, \omega, D} \\
\leq & \left\{\left(\frac{1}{8 \delta^{2}}\right)^{q}(\omega R)^{q+1} \mathrm{e}^{\frac{\omega R}{2}} \frac{p^{p}}{(q !)^{2}(q+1) !}\right\} \\
& \cdot\left\{\frac{2^{L+1}}{\sqrt{\pi} \rho^{L-K+1}} \omega^{-K} h^{-1}\left(1+(\omega h)^{-L+K}\right) \sqrt{L+1}(L-K+1) !\right\}\|P\|_{K, \omega, D} \\
\leq & \frac{2}{\sqrt{\pi} \rho^{L-K+1}}\left(\frac{1}{8 \delta^{2}}\right)^{q}\left(2^{L} \sqrt{L+1}\right) \\
& \cdot(\omega R)^{q+1-K}\left(1+(\omega h)^{-L+K}\right) \mathrm{e}^{\frac{\omega R}{2}} \frac{R^{K}}{h} \frac{p^{p}\left\lfloor\frac{q+1}{2}\right\rfloor !}{(q !)^{2}(q+1) !}\|P\|_{K, \omega, D} \cdot
\end{aligned}
$$

From Stirling's formula, we infer

$$
\sqrt{2 \pi} \sqrt{n} n^{n} \mathrm{e}^{-n} \mathrm{e}^{\frac{1}{12 n+1}}<n !<\sqrt{2 \pi} \sqrt{n} n^{n} \mathrm{e}^{-n} \mathrm{e}^{\frac{1}{12 n}}, \quad n \geq 1 .
$$


We use this to bound

$$
\begin{aligned}
\frac{p^{p}\left\lfloor\frac{q+1}{2}\right\rfloor !}{(q !)^{2}(q+1) !} & \leq \frac{(2 q+2)^{2 q+1}\left\lfloor\frac{q+1}{2}\right\rfloor !}{((q+1) !)^{3}}(q+1)^{2} \\
& <\frac{2^{2 q+1}}{2 \pi} \frac{(q+1)^{2 q+3}\left(\frac{q+1}{2}\right)^{\left(\frac{q+1}{2}\right)+\frac{1}{2}}}{(q+1)^{3(q+1)+\frac{3}{2}}} \mathrm{e}^{3(q+1)-\frac{q}{2}} \mathrm{e}^{-\frac{3}{12(q+1)+1}+\frac{1}{6 q}} .
\end{aligned}
$$

For $q \geq 3$, since the exponent in the last factor on the right-hand side of the last inequality is negative, we get

$$
\frac{p^{p}\left\lfloor\frac{q+1}{2}\right\rfloor !}{(q !)^{2}(q+1) !} \leq \frac{\mathrm{e}^{3}}{2 \pi}\left(2 \sqrt{2} \mathrm{e}^{\frac{5}{2}}\right)^{q}(q+1)^{-\frac{q+1}{2}} .
$$

For $q=1,2$, one can see directly that the same bound holds true, thus we can use it for any $q \geq 1$ and obtain

$$
\begin{aligned}
\left\|V_{1}[P]-\sum_{k=-q}^{q} \alpha_{k} \mathrm{e}^{i \omega \boldsymbol{x} \cdot \boldsymbol{d}_{k}}\right\|_{L^{\infty}\left(B_{R}\right)} \leq & \frac{\mathrm{e}^{3}}{\pi^{\frac{3}{2}} \rho^{L-K+1}}\left(\frac{\mathrm{e}^{\frac{5}{2}}}{2 \sqrt{2} \delta^{2}}\right)^{q}\left(2^{L} \sqrt{L+1}\right) \\
& \cdot(\omega R)^{q+1-K}\left(1+(\omega h)^{-L+K}\right) \mathrm{e}^{\frac{\omega R}{2}} \frac{R^{K}}{h} \frac{1}{(q+1)^{\frac{q+1}{2}}}\|P\|_{K, \omega, D} ;
\end{aligned}
$$

this concludes the proof.

In Sect. 5, we will use the bound in Lemma 4.3 with $R=h$ in the derivation of $h p$-approximation error estimates of Helmholtz solutions by plane waves in the 2D case (see Theorem 5.2).

Remark 4.4. Notice that, in Lemma 4.3, the assumption (19), which basically means $L \lesssim q / 2$, has been used only once, i.e., in the inequalities chain (28).

We could modify the condition (19) into $L-K \leq \eta(q-1), \eta \in(0,1)$. This allows to choose higher order generalized harmonic polynomials in the final $p$-estimate and modify the constants in Theorem 5.2 and in Corollary 5.5. However, this does not affect the general order of convergence.

\subsection{The three-dimensional case}

Now, we would like to prove an approximation estimate similar to Lemma 4.3 in a three-dimensional setting. The two-dimensional case has shown that the proof of the order of convergence with respect to $q$ requires a sharp bound on the norm of the inverse of the matrix $\boldsymbol{A}$. In three-dimensions, the corresponding matrix is $\boldsymbol{M}$, defined in (16). This matrix is more complicated and it is not of Vandermonde type. As a consequence, we are not able to bound the norm of $\boldsymbol{M}^{-1}$ with a reasonable dependence on $q$ in the general case, but we restrict ourselves to a particular choice of the directions $\boldsymbol{d}_{l, m}$.

Lemma 4.5. Given $q \in \mathbb{N}$, there exists a set of directions $\left\{\boldsymbol{d}_{l, m}\right\}_{0 \leq|m| \leq l \leq q} \subset \mathbb{S}^{2}$ such that

$$
\left\|\boldsymbol{M}^{-1}\right\|_{1} \leq 2 \sqrt{\pi} p=2 \sqrt{\pi}(q+1)^{2} .
$$

Proof. Given a set of $p=(q+1)^{2}$ directions $\left\{\boldsymbol{d}_{l, m}\right\}$, we define the determinant

$$
\Delta:\left(\mathbb{S}^{2}\right)^{p} \mapsto \mathbb{C}, \quad \Delta\left(\left\{\boldsymbol{d}_{l, m}\right\}\right):=\operatorname{det}(\boldsymbol{M}) .
$$

This is a continuous function, so $|\Delta(\cdot)|$ achieves its maximum in, say,

$$
\left\{\boldsymbol{d}_{l, m}^{*}\right\}_{0 \leq|m| \leq l \leq q} \in\left(\mathbb{S}^{2}\right)^{p} .
$$


Thanks to Lemma $4.2, \Delta(\cdot)$ is not identically zero, so it is possible to define the polynomials

$$
L_{l, m}(\boldsymbol{x}):=\frac{\left.\Delta\left(\boldsymbol{d}_{0,0}^{*}, \ldots, \boldsymbol{x}, \ldots, \boldsymbol{d}_{q, q}^{*}\right\}\right)}{\Delta\left(\left\{\boldsymbol{d}_{l, m}^{*}\right\}\right)}, \quad \boldsymbol{x} \in \mathbb{S}^{2}
$$

(in the numerator, the direction $\boldsymbol{d}_{l, m}^{*}$ is replaced by $\boldsymbol{x}$ ). From their definition, it is clear that these functions are spherical polynomials of degree at most $q$; they satisfy

$$
\begin{array}{ll}
L_{l, m}\left(\boldsymbol{d}_{l^{\prime}, m^{\prime}}^{*}\right)=\delta_{l, l^{\prime}} \delta_{m, m^{\prime}}, & 0 \leq|m| \leq l \leq q \\
& 0 \leq\left|m^{\prime}\right| \leq l^{\prime} \leq q
\end{array}
$$

which means that they are the Lagrange polynomials of the set $\left\{\boldsymbol{d}_{l, m}^{*}\right\}$ and

$$
\left\|L_{l, m}\right\|_{L^{\infty}\left(\mathbb{S}^{2}\right)}=1 .
$$

Now, we show that the set $\left\{\boldsymbol{d}_{l, m}^{*}\right\}$ is the one which satisfies (30). With the choice $\boldsymbol{d}_{l, m}=\boldsymbol{d}_{l, m}^{*}$, the entries of $M^{-1}$ satisfy

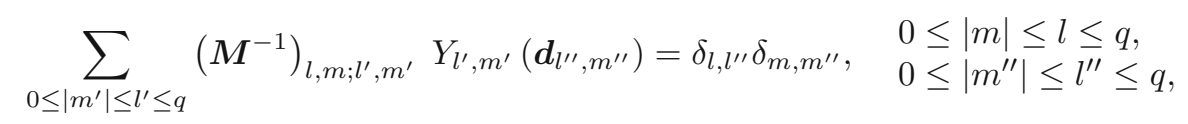

which means $\left(\boldsymbol{M}^{-1}\right)_{l, m ; l^{\prime}, m^{\prime}}$ is the $\left(l^{\prime}, m^{\prime}\right)$ th coefficient of $L_{l, m}$ with respect to the standard spherical harmonic basis. This gives:

$$
\begin{aligned}
\left\|\boldsymbol{M}^{-1}\right\|_{1} & =\max _{0 \leq\left|m^{\prime}\right| \leq l^{\prime} \leq q} \sum_{0 \leq|m| \leq l \leq q}\left|\left(\boldsymbol{M}^{-1}\right)_{l, m ; l^{\prime}, m^{\prime}}\right| \\
& \leq p \max _{0 \leq\left|m^{\prime}\right| \leq l^{\prime} \leq q} \max _{0 \leq|m| \leq l \leq q}\left|\left(\boldsymbol{M}^{-1}\right)_{l, m ; l^{\prime}, m^{\prime}}\right| \\
& \leq p \max _{0 \leq|m| \leq l \leq q}\left(\sum_{0 \leq\left|m^{\prime}\right| \leq l^{\prime} \leq q}\left|\left(\boldsymbol{M}^{-1}\right)_{l, m ; l^{\prime}, m^{\prime}}\right|^{2}\right)^{\frac{1}{2}} \\
& =p \max _{0 \leq|m| \leq l \leq q}\left\|L_{l, m}\right\|_{L^{2}\left(\mathbb{S}^{2}\right)} \\
& \leq p \sqrt{4 \pi} \max _{0 \leq|m| \leq l \leq q}\left\|L_{l, m}\right\|_{L^{\infty}\left(\mathbb{S}^{2}\right)}=2 \sqrt{\pi} p,
\end{aligned}
$$

where we used the orthonormality of the spherical harmonics in $L^{2}\left(\mathbb{S}^{2}\right)$.

The first part of this proof is adapted from that of [21, Theorem 14.1], which is a special case of the Auerbach theorem.

Remark 4.6. Lemma 4.5 does not provide a way of computing the set of directions satisfying (30). However, an efficient algorithm that computes systems of directions which satisfy a bound close to (30) is introduced in [22]. The computed directions can be downloaded from the Web site [24]. The table presented on that Web site shows that the Lebesgue constant for $p=(q+1)^{2}$ computed directions is smaller than $2 q$, which gives the slightly worse bound $\left\|M^{-1}\right\|_{1} \leq 4 \sqrt{\pi} p q$.

Now, we can prove the three-dimensional counterpart of Lemma 4.3. 
Lemma 4.7. Let $D \subset \mathbb{R}^{3}$ be a domain that satisfies Assumption 2.1, $q \in \mathbb{N}, p=(q+1)^{2}$, and let $\left\{\boldsymbol{d}_{l, m}\right\}_{0 \leq|m| \leq l \leq q} \subset \mathbb{S}^{2}$ be a set of directions for which the matrix $\boldsymbol{M}$ is invertible. Then, for every harmonic polynomial $P$ of degree $L \leq q$ and for every $R>0$ and $K \in \mathbb{N}$ satisfying

$$
0 \leq K \leq L \leq q, \quad L-K \leq\left\lfloor\frac{q-1}{2}\right\rfloor
$$

there exists a vector $\vec{\alpha} \in \mathbb{C}^{p}$, such that

$$
\left\|V_{1}[P]-\sum_{\substack{l=0, \ldots, q ; \\|m| \leq l}} \alpha_{l, m} \mathrm{e}^{i \omega \boldsymbol{x} \cdot \boldsymbol{d}_{l, m}}\right\|\left\|_{L^{\infty}\left(B_{R}\right)} \leq C(\omega, \rho, h, R, q, K, L)\right\| \boldsymbol{M}^{-1}\left\|_{1}\right\| P \|_{K, \omega, D},
$$

where

$$
\begin{aligned}
C(\omega, \rho, h, R, q, K, L)= & \frac{1}{\rho^{L-K+\frac{3}{2}}} \frac{(L+1)^{2} \mathrm{e}^{K+1}}{\sqrt{2}^{L}} \\
& \cdot(\omega R)^{q+1-K} \frac{R^{K}}{h^{\frac{3}{2}}}\left(1+(\omega h)^{-L+K}\right) \mathrm{e}^{\frac{\omega R}{2}} \frac{1}{q^{\frac{q-3}{2}}} \frac{1}{2 \sqrt{\pi}(q+1)^{2}} .
\end{aligned}
$$

Proof. As in two-dimensions, we write the polynomial

$$
P(\boldsymbol{x})=\sum_{l=0}^{L} \sum_{m=-l}^{l} a_{l, m}|\boldsymbol{x}|^{l} Y_{l, m}\left(\frac{\boldsymbol{x}}{|\boldsymbol{x}|}\right)
$$

and we use the Jacobi-Anger expansion:

$$
\begin{aligned}
& V_{1}[P](\boldsymbol{x})-\sum_{\substack{l^{\prime}=0, \ldots, q ; \\
\left|m^{\prime}\right| \leq l^{\prime}}} \alpha_{l^{\prime}, m^{\prime}} \mathrm{e}^{i \omega \boldsymbol{x} \cdot \boldsymbol{d}_{l^{\prime}, m^{\prime}}} \\
& \stackrel{(9),(14)}{=} \sum_{l=0}^{L} \sum_{m=-l}^{l} a_{l, m}\left(\frac{1}{2 \omega}\right)^{l} \frac{(2 l+1) !}{l !} Y_{l, m}\left(\frac{\boldsymbol{x}}{|\boldsymbol{x}|}\right) j_{l}(\omega|\boldsymbol{x}|) \\
& -4 \pi \sum_{l \geq 0} i^{l} j_{l}(\omega|\boldsymbol{x}|) \sum_{m=-l}^{l} Y_{l, m}\left(\frac{\boldsymbol{x}}{|\boldsymbol{x}|}\right) \sum_{\substack{l^{\prime}=0, \ldots, q ; \\
\left|m^{\prime}\right| \leq l^{\prime}}} \alpha_{l^{\prime}, m^{\prime}} \overline{Y_{l, m}\left(\boldsymbol{d}_{l^{\prime}, m^{\prime}}\right)} \\
& =-4 \pi \sum_{l \geq q+1} i^{l} j_{l}(\omega|\boldsymbol{x}|) \sum_{m=-l}^{l} Y_{l, m}\left(\frac{\boldsymbol{x}}{|\boldsymbol{x}|} \sum_{\substack{\sum^{\prime}=0, \ldots, q ; \\
\left|m^{\prime}\right| \leq l^{\prime}}}^{\substack{l \\
l}} \alpha_{l^{\prime}, m^{\prime}} \overline{Y_{l, m}\left(\boldsymbol{d}_{l^{\prime}, m^{\prime}}\right)}\right.
\end{aligned}
$$

provided that the vector $\vec{\alpha} \in \mathbb{C}^{p}$ is the solution of the linear system $\boldsymbol{M} \cdot \vec{\alpha}=\vec{\beta}$ with

$$
\beta_{l, m}= \begin{cases}\frac{1}{4 \pi}\left(\frac{1}{2 i \omega}\right)^{l} \frac{(2 l+1) !}{l !} a_{l, m}, & l=0, \ldots, L ;|m| \leq l \\ 0, & l=L+1, \ldots, q ;|m| \leq l\end{cases}
$$

and $\boldsymbol{M}$ is the $p \times p$ matrix defined in (16). 
Now, we can bound the coefficients $a_{l, m}$ with the norms of the polynomial $P$, denoting $r=|\boldsymbol{x}|$ :

$$
\begin{aligned}
|P|_{\mu, D}^{2} \geq & \left\|\frac{\partial^{\mu}}{\partial r^{\mu}} P\right\|_{0, B_{\rho h}}^{2}=\left\|\sum_{l=\mu}^{L} \sum_{m=-l}^{l} a_{l, m} \frac{l !}{(l-\mu) !} r^{l-\mu} Y_{l, m}\left(\frac{\boldsymbol{x}}{|\boldsymbol{x}|}\right)\right\|_{0, B_{\rho h}}^{2} \\
= & \int_{0}^{\rho h} \sum_{l=\mu}^{L} \sum_{m=-l}^{l} \sum_{l^{\prime}=\mu}^{L} \sum_{m^{\prime}=-l^{\prime}}^{l^{\prime}} a_{l, m} \overline{a_{l^{\prime}, m^{\prime}}} \frac{l ! l^{\prime} !}{(l-\mu) !\left(l^{\prime}-\mu\right) !} r^{l+l^{\prime}-2 \mu} \\
& \cdot \int_{\mathbb{S}^{2}} Y_{l, m}(\boldsymbol{d}) \overline{Y_{l^{\prime}, m^{\prime}}(\boldsymbol{d})} \mathrm{d} \boldsymbol{d} \quad r^{2} \mathrm{~d} r \\
= & \sum_{l=\mu}^{L} \sum_{m=-l}^{l}\left|a_{l, m}\right|^{2} \frac{(l !)^{2}}{((l-\mu) !)^{2}} \frac{(\rho h)^{2(l-\mu)+3}}{2(l-\mu)+3} \quad 0 \leq \mu \leq L,
\end{aligned}
$$

thanks to the orthonormality of the spherical harmonics. Choosing $\mu=l$ and $\mu=K$, this gives:

$$
\begin{aligned}
\sum_{m=-l}^{l}\left|a_{l, m}\right| & \leq \sqrt{2 l+1}\left(\sum_{m=-l}^{l}\left|a_{l, m}\right|^{2}\right)^{\frac{1}{2}} \leq \sqrt{2 l+1} \frac{\sqrt{3}}{l !(\rho h)^{\frac{3}{2}}}|P|_{l, D} \quad 0 \leq l \leq L, \\
\sum_{m=-l}^{l}\left|a_{l, m}\right| & \leq \sqrt{2 l+1} \frac{(l-K) ! \sqrt{2(l-K)+3}}{l !(\rho h)^{l-K+\frac{3}{2}}}|P|_{K, D} \\
& \leq \frac{(l-K) !(2 l+2)}{l !(\rho h)^{l-K+\frac{3}{2}}}|P|_{K, D} \quad K \leq l \leq L .
\end{aligned}
$$

Now, for every $\boldsymbol{d}_{l^{\prime}, m^{\prime}}$ and for every $\boldsymbol{x} \in B_{R}$, we have

$$
\begin{aligned}
& \left|4 \pi \sum_{l \geq q+1} i^{l} j_{l}(\omega|\boldsymbol{x}|) \sum_{m=-l}^{l} Y_{l, m}\left(\frac{\boldsymbol{x}}{|\boldsymbol{x}|}\right) \overline{Y_{l, m}\left(\boldsymbol{d}_{l^{\prime}, m^{\prime}}\right)}\right| \\
& \leq 4 \pi \sum_{l \geq q+1} \sqrt{\frac{\pi}{2 \omega|\boldsymbol{x}|}}\left|J_{l+\frac{1}{2}}(\omega|\boldsymbol{x}|)\right| \sqrt{\sum_{m=-l}^{l}\left|Y_{l, m}\left(\frac{\boldsymbol{x}}{|\boldsymbol{x}|}\right)\right|^{2}} \sqrt{\sum_{m=-l}^{l}\left|Y_{l, m}\left(\boldsymbol{d}_{l^{\prime}, m^{\prime}}\right)\right|^{2}} \\
& \stackrel{(23)}{\leq} 4 \pi \sqrt{\frac{\pi}{2 \omega|\boldsymbol{x}|}} \sum_{l \geq q+1} \frac{(\omega|\boldsymbol{x}|)^{l+\frac{1}{2}}}{\Gamma\left(l+\frac{3}{2}\right) 2^{l+\frac{1}{2}}} \frac{2 l+1}{4 \pi} \\
& \stackrel{j=l-q-1}{\leq} \frac{\sqrt{\pi}}{2}\left(\frac{\omega|\boldsymbol{x}|}{2}\right)^{q+1} \sum_{j=0}^{\infty} \frac{\left(\frac{\omega|\boldsymbol{x}|}{2}\right)^{j} 2\left(q+j+1+\frac{1}{2}\right)}{\Gamma\left(q+j+1+\frac{3}{2}\right)} \\
& \leq \sqrt{\pi}\left(\frac{\omega|\boldsymbol{x}|}{2}\right)^{q+1} \frac{q ! 2^{2 q+1}}{\sqrt{\pi}(2 q+1) !} \sum_{j=0}^{\infty} \frac{\left(\frac{\omega|\boldsymbol{x}|}{2}\right)^{j}}{j !} \leq \frac{q ! 2^{q}}{(2 q+1) !}(\omega R)^{q+1} \mathrm{e}^{\frac{\omega R}{2}},
\end{aligned}
$$

where in the second inequality, we have bounded the sum of the spherical harmonics with (2.4.105) of [20], and in the fourth inequality, we have used

$$
\frac{\left(q+j+\frac{3}{2}\right)}{\Gamma\left(q+j+1+\frac{3}{2}\right)}=\frac{1}{\Gamma\left(q+j+\frac{3}{2}\right)} \leq \frac{1}{\Gamma\left(q+\frac{3}{2}\right) \Gamma(j+1)}=\frac{q ! 2^{2 q+1}}{\sqrt{\pi}(2 q+1) ! j !} .
$$


We will also need the following bound. When $q \geq 3$, using the Stirling formula (29), $e<2 \sqrt{2}$ and the hypothesis on the indices, we have

$$
\begin{aligned}
\frac{(L-K) !}{2^{q-L} q !} & \leq \frac{(L-K)^{L-K+\frac{1}{2}} \mathrm{e}^{q+1}}{2^{q-L} q^{q+\frac{1}{2}} \mathrm{e}^{L-K}} \\
& \stackrel{(31)}{\leq} \mathrm{e}^{K+1}\left(\frac{e}{2}\right)^{q-L} \frac{\left\lfloor\frac{q-1}{2}\right\rfloor^{\left\lfloor\frac{q-1}{2}\right\rfloor+\frac{1}{2}}}{q^{q+\frac{1}{2}}} \\
& \leq \sqrt{2}^{-L} \mathrm{e}^{K+1}\left(\frac{e}{2 \sqrt{2}}\right)^{q-L} \frac{(q-1)^{\frac{q}{2}}}{q^{q+\frac{1}{2}}} \\
& \leq \sqrt{2}^{-L} \mathrm{e}^{K+1} q^{-\frac{q}{2}+\frac{3}{2}} \frac{1}{(q+1)^{2}} .
\end{aligned}
$$

The same bound holds true also for $q=1,2$.

We plug (36) in (33) with the definition of $\vec{\beta}$ and the bound (35) on the coefficients $a_{l, m}$ with $K=l$ and obtain

$$
\begin{aligned}
& \left\|V_{1}[P]-\sum_{\substack{l=0, \ldots, q ; \\
|m| \leq l}} \alpha_{l, m} \mathrm{e}^{i \omega \boldsymbol{x} \cdot \boldsymbol{d}_{l, m}}\right\|_{L^{\infty}\left(B_{R}\right)} \\
& \stackrel{(33)}{\leq} \sup _{\substack{\boldsymbol{x} \in B_{R} \\
l^{\prime}=0, \ldots \ldots, q, m^{\prime}=-l^{\prime}, \ldots, l^{\prime}}}\left|4 \pi \sum_{l \geq q+1} i^{l} j_{l}(\omega|\boldsymbol{x}|) \sum_{m=-l}^{l} Y_{l, m}\left(\frac{\boldsymbol{x}}{|\boldsymbol{x}|}\right) \overline{Y_{l, m}\left(\boldsymbol{d}_{l^{\prime}, m^{\prime}}\right)}\right| \cdot\|\vec{\alpha}\|_{1} \\
& \stackrel{(36)}{\leq} \frac{q ! 2^{q}}{(2 q+1) !}(\omega R)^{q+1} \mathrm{e}^{\frac{\omega R}{2}}\left\|\boldsymbol{M}^{-1}\right\|_{1}\|\vec{\beta}\|_{1} \\
& \stackrel{(34)}{\leq}\left\|M^{-1}\right\|_{1} \frac{q ! 2^{q}}{(2 q+1) !}(\omega R)^{q+1} \mathrm{e}^{\frac{\omega R}{2}} \sum_{l=0}^{L} \sum_{m=-l}^{l} \frac{1}{4 \pi}\left(\frac{1}{2 \omega}\right)^{l} \frac{(2 l+1) !}{l !}\left|a_{l, m}\right| \\
& \stackrel{(35)}{\leq} \frac{\left\|\boldsymbol{M}^{-1}\right\|_{1}}{4 \pi} \frac{q ! 2^{q}}{(2 q+1) !}(\omega R)^{q+1} \mathrm{e}^{\frac{\omega R}{2}}\left[\sum_{l=0}^{K-1}\left(\frac{1}{2 \omega}\right)^{l} \frac{(2 l+1) ! \sqrt{3} \sqrt{2 l+1}}{l ! l !(\rho h)^{\frac{3}{2}}}|P|_{l, D}\right. \\
& \left.+\sum_{l=K}^{L}\left(\frac{1}{2 \omega}\right)^{l} \frac{(2 l+1) !(l-K) !(2 l+2)}{l ! l !(\rho h)^{l-K+\frac{3}{2}}}|P|_{K, D}\right] \\
& \leq \sqrt{\frac{3}{\pi}} \frac{\left\|M^{-1}\right\|_{1}}{4 \sqrt{\pi}} \frac{q ! 2^{q}}{\rho^{L-K+\frac{3}{2}}(2 q+1) !} \frac{(\omega R)^{q+1}}{h^{\frac{3}{2}}} \mathrm{e}^{\frac{\omega R}{2}}\left[\sum_{l=0}^{K-1} \frac{(2 l+1) ! \sqrt{2 l+1}}{2^{l} l ! l !}\right. \\
& \left.+\sum_{l=K}^{L} \frac{(2 l+1) !(l-K) !(2 l+2)}{2^{l} l ! l !(\omega h)^{l-K}}\right] \omega^{-K}\|P\|_{K,, \omega, D} \\
& \leq \frac{\left\|\boldsymbol{M}^{-1}\right\|_{1}}{4 \sqrt{\pi}} \frac{1}{\rho^{L-K+\frac{3}{2}}} \frac{q ! 2^{q}}{(2 q+1) !}\left[\frac{(2 L+1) !}{2^{L} L ! L !}((L+1)(L-K) !(2 L+2))\right] \\
& \cdot(\omega R)^{q+1-K} \frac{R^{K}}{h^{\frac{3}{2}}}\left(1+(\omega h)^{-L+K}\right) \mathrm{e}^{\frac{\omega R}{2}}\|P\|_{K, \omega, D}
\end{aligned}
$$




$$
\begin{aligned}
\leq & \frac{\left\|\boldsymbol{M}^{-1}\right\|_{1}}{2 \sqrt{\pi}} \frac{1}{\rho^{L-K+\frac{3}{2}}} \frac{1}{q ! 2^{q}} \frac{q ! q ! 4^{q}}{(2 q+1) !} \frac{(2 L+1) !}{4^{L} L ! L !} 2^{L}(L+1)^{2}(L-K) ! \\
& \cdot(\omega R)^{q+1-K} \frac{R^{K}}{h^{\frac{3}{2}}}\left(1+(\omega h)^{-L+K}\right) \mathrm{e}^{\frac{\omega R}{2}}\|P\|_{K, \omega, D} \\
\leq & \frac{\left\|\boldsymbol{M}^{-1}\right\|_{1}}{2 \sqrt{\pi}} \frac{1}{\rho^{L-K+\frac{3}{2}}} \frac{(L-K) !}{q ! 2^{q-L}}(L+1)^{2} \\
& \cdot(\omega R)^{q+1-K} \frac{R^{K}}{h^{\frac{3}{2}}}\left(1+(\omega h)^{-L+K}\right) \mathrm{e}^{\frac{\omega R}{2}}\|P\|_{K, \omega, D} \\
& \leq \frac{\left\|M^{-1}\right\|_{1}}{2 \sqrt{\pi}(q+1)^{2}} \frac{(L+1)^{2} \mathrm{e}^{K+1}}{\rho^{L-K+\frac{3}{2}} \sqrt{2}^{L}} q^{-\frac{q}{2}+\frac{3}{2}} \\
& \cdot(\omega R)^{q+1-K} \frac{R^{K}}{h^{\frac{3}{2}}}\left(1+(\omega h)^{-L+K}\right) \mathrm{e}^{\frac{\omega R}{2}}\|P\|_{K, \omega, D},
\end{aligned}
$$

where we have used the monotonicity of the increasing sequences $l \mapsto \frac{(2 l+1) !}{2^{l} l ! l !}$ and $l \mapsto \frac{(2 l+1) !}{4^{l} l ! l !}=\frac{2 \Gamma(l+3 / 2)}{\sqrt{\pi} \Gamma(l+1)}$.

Combining Lemmas 4.7 and 4.5 immediately gives the following result.

Corollary 4.8. Let $D \subset \mathbb{R}^{3}$ be a domain that satisfies Assumption $2.1, q \in \mathbb{N}$ and $p=(q+1)^{2}$. Then, there exists a set of directions $\left\{\boldsymbol{d}_{l, m}\right\}_{0 \leq|m| \leq l \leq q} \subset \mathbb{S}^{2}$ such that for every harmonic polynomial $P$ of degree $L \leq q$ and for every $R>0$ and $K \in \mathbb{N}$ satisfying (31), there exists a vector $\vec{\alpha} \in \mathbb{C}^{p}$ such that

$$
\left\|V_{1}[P]-\sum_{\substack{l=0, \ldots, q ; \\|m| \leq l}} \alpha_{l, m} \mathrm{e}^{i \omega \boldsymbol{x} \cdot \boldsymbol{d}_{l, m}}\right\|_{L^{\infty}\left(B_{R}\right)} \leq C(\omega, \rho, h, R, q, K, L)\|P\|_{K, \omega, D},
$$

where

$$
\begin{aligned}
C(\omega, \rho, h, R, q, K, L)= & \frac{1}{\rho^{L-K+\frac{3}{2}}} \frac{(L+1)^{2} \mathrm{e}^{K+1}}{\sqrt{2}^{L}} \\
& \cdot(\omega R)^{q+1-K} \frac{R^{K}}{h^{\frac{3}{2}}}\left(1+(\omega h)^{-L+K}\right) \mathrm{e}^{\frac{\omega R}{2}} \frac{1}{q^{\frac{q-3}{2}}} .
\end{aligned}
$$

Lemma 4.7 provides a way to compute a plane wave approximation of a given generalized harmonic polynomial. Solving the linear system $\boldsymbol{M} \cdot \vec{\alpha}=\vec{\beta}$, with the matrix $M$ defined in (16) and the right-hand side $\vec{\beta}$ as in (34), gives the coefficient vector $\vec{\alpha}$ of the approximating linear combination of plane waves. Since $M$ is independent of $\omega$ and $h$, the conditioning of this problem depends only on the choice of the directions. Hence, in terms of stability, approximation with plane waves is no less stable with respect to $\omega$ than approximation by generalized harmonic polynomials.

\section{Approximation of Helmholtz solutions by plane waves}

In order to use Lemmas 4.3 and 4.7 to derive error estimates for the approximation of homogeneous Helmholtz solutions in $P W_{\omega, p}\left(\mathbb{R}^{N}\right)$, we need to link the Sobolev norms to the $L^{\infty}$ norm of the error. This is done in the following lemma that generalizes the usual Cauchy estimates for harmonic functions to the Helmholtz case. The result is a simple consequence of the continuity of the Vekua transform. 
Lemma 5.1. Let $D \subset \mathbb{R}^{N}, N=2,3$ be a domain as in Assumption 2.1, and let $u \in H^{j}\left(B_{h}\right), j \in \mathbb{N}$ be a solution to the homogeneous Helmholtz equation with $\omega>0$. Then, we have

$$
\|u\|_{j, \omega, D} \leq C_{N, j} \rho^{\frac{1-N}{2}-j}\left(1+(\omega h)^{j+4}\right) \mathrm{e}^{\frac{1}{2} \omega h} h^{\frac{N}{2}-j}\|u\|_{L^{\infty}\left(B_{h}\right)} .
$$

where the constant $C$ depends only on $N$ and $j$.

Proof. Assumption 2.1 implies that $D \subset B_{(1-\rho) h}$ and henceforth $d\left(D, \partial B_{h}\right) \geq \rho h$. Using the Cauchy estimates for harmonic functions and the continuity of the Vekua operators, we have

$$
\begin{aligned}
\|u\|_{j, \omega, D} & \stackrel{(4)}{\leq} C_{N} \rho^{\frac{1-N}{2}}(1+j)^{\frac{3}{2} N+\frac{1}{2}} \mathrm{e}^{j}\left(1+(\omega h)^{2}\right)\left\|V_{2}[u]\right\|_{j, \omega, D} \\
& \leq C_{N, j} \rho^{\frac{1-N}{2}}\left(1+(\omega h)^{2}\right) \sum_{l=0}^{j} \omega^{j-l}\left|V_{2}[u]\right|_{l, D} \\
& \leq C_{N, j} \rho^{\frac{1-N}{2}}\left(1+(\omega h)^{2}\right) \sum_{l=0}^{j} \omega^{j-l} h^{\frac{N}{2}}\left|V_{2}[u]\right|_{W^{l, \infty}(D)} \\
& \leq C_{N, j} \rho^{\frac{1-N}{2}}\left(1+(\omega h)^{2}\right) \sum_{l=0}^{j} \omega^{j-l} h^{\frac{N}{2}}(\rho h)^{-l}\left\|V_{2}[u]\right\|_{L^{\infty}\left(B_{h}\right)} \\
& \leq C_{N, j} \rho^{\frac{1-N}{2}-j}\left(1+(\omega h)^{j+2}\right) h^{\frac{N}{2}-j}\left\|V_{2}[u]\right\|_{L^{\infty}\left(B_{h}\right)} \\
& \text { (7) on } B_{h} \\
& \leq C_{N, j} \rho^{\frac{1-N}{2}-j}\left(1+(\omega h)^{j+4}\right) \mathrm{e}^{\frac{1}{2} \omega h} h^{\frac{N}{2}-j}\|u\|_{L^{\infty}\left(B_{h}\right)},
\end{aligned}
$$

where in the 4 th inequality, we have used [9, Theorem 2.10] to bound the $W^{1, \infty}$-seminorm on $D$ by the $L^{\infty}$-norm on $B_{h}$ and, in the last step, the exponential has coefficient $1 / 2$ because the ball $B_{h}$ has diameter $2 h$ and shape parameter $\rho\left(B_{h}\right)=1 / 2$.

Now, we can state the main results: the $h p$-approximation estimates for homogeneous Helmholtz solutions in $H^{j}(D)$ with plane waves in $P W_{\omega, p}(D)$. We consider the two cases $N=2$ and $N=3$ separately in Theorems 5.2 and 5.3, respectively; we will write a simpler (and probably more useful) version in Corollary 5.5.

Theorem 5.2. (hp-estimates, $N=2$ ) Let $u \in H^{K+1}(D)$ be a solution of the homogeneous Helmholtz equation in a domain $D \subset \mathbb{R}^{2}$ satisfying Assumption 2.1 and the exterior cone condition with angle $\lambda \pi$ (see Definition 3.1). Fix $q \geq 1$, set $p=2 q+1$ and let the directions $\left\{\boldsymbol{d}_{k}=\left(\cos \theta_{k}, \sin \theta_{k}\right)\right\}_{k=-q, \ldots, q}$ satisfy the condition (18).

Then, for every integer $L$ satisfying

$$
0 \leq K \leq L \leq q, \quad L-K \leq\left\lfloor\frac{q-1}{2}\right\rfloor,
$$

there exists $\vec{\alpha} \in \mathbb{C}^{p}$ such that, for every $0 \leq j \leq K$,

$$
\begin{aligned}
& \left\|u-\sum_{k=1}^{p} \alpha_{k} \mathrm{e}^{i \omega \boldsymbol{x} \cdot \boldsymbol{d}_{k}}\right\|_{j, \omega, D} \leq C \mathrm{e}^{\left(\frac{7}{4}-\frac{3}{4} \rho\right) \omega h}\left(1+(\omega h)^{q+j-K+8}\right) h^{K+1-j} \\
& \quad\left\{\left(\frac{\log (L+2)}{L+2}\right)^{\lambda(K+1-j)}+\left(\frac{2}{\rho}\right)^{L} \sqrt{\frac{L+1}{q+1}}\left(\frac{\mathrm{e}^{\frac{5}{2}}}{2 \sqrt{2} \delta^{2} \sqrt{q+1}}\right)^{q}\right\}\|u\|_{K+1, \omega, D},
\end{aligned}
$$

where the constant $C>0$ depends only on $j, K$ and the shape of $D$, but is independent of $q, L, \delta, \omega, h$ and $u$.

Proof. Let $Q$ be the generalized harmonic polynomial of degree at most $L$ equal to $Q_{L}^{\prime}$ from Theorem 3.2, item (i). 
Since $V_{2}[Q]$ approximates $V_{2}[u]$, we notice that, for $K \geq 1$,

$$
\begin{aligned}
\left\|V_{2}[Q]\right\|_{K, \omega, D} & \leq\left\|V_{2}[u]\right\|_{K, \omega, D}+\left\|V_{2}[u]-V_{2}[Q]\right\|_{K, \omega, D} \\
& \stackrel{(55)}{\leq}(1+C)\left\|V_{2}[u]\right\|_{K, \omega, D} \\
& \stackrel{(6)}{\leq} C\left(1+(\omega h)^{4}\right) \mathrm{e}^{\frac{3}{4}(1-\rho) \omega h}\|u\|_{K, \omega, D},
\end{aligned}
$$

where $C$ depends only on $K$ and the shape of $D$. In the second step, we could use the stability bound (55) with $j=k+1=K$ and $\phi=V_{2}[u]$ because $Q=Q_{L}^{\prime}=V_{1}[P]$, with $P$ from Theorem A.3.

We combine all the ingredients and obtain, in the case $K \geq 1$,

$$
\begin{aligned}
\left\|u-\sum_{k=1}^{p} \alpha_{k} \mathrm{e}^{i \omega \boldsymbol{x} \cdot \boldsymbol{d}_{k}}\right\|_{j, \omega, D} \leq & \|u-Q\|_{j, \omega, D}+\left\|Q-\sum_{k=1}^{p} \alpha_{k} \mathrm{e}^{i \omega \boldsymbol{x} \cdot \boldsymbol{d}_{k}}\right\|_{j, \omega, D} \\
\underbrace{}_{(10),(39)} & C\left(1+(\omega h)^{j+6}\right) \mathrm{e}^{\frac{3}{4}(1-\rho) \omega h}\left(\frac{\log (L+2)}{L+2}\right)^{\lambda(K+1-j)} h^{K+1-j}\|u\|_{K+1, \omega, D} \\
& +C\left(1+(\omega h)^{j+4}\right) \mathrm{e}^{\frac{1}{2} \omega h} h^{1-j}\left\|Q-\sum_{k=1}^{p} \alpha_{k} \mathrm{e}^{i \omega \boldsymbol{x} \cdot \boldsymbol{d}_{k}}\right\|_{L^{\infty}\left(B_{h}\right)} \\
\leq & C\left(1+(\omega h)^{j+6}\right) \mathrm{e}^{\frac{3}{4}(1-\rho) \omega h}\left(\frac{\log (L+2)}{L+2}\right)^{\lambda(K+1-j)} h^{K+1-j}\|u\|_{K+1, \omega, D} \\
& +C \rho^{-L+K-1}\left(\frac{\mathrm{e}^{\frac{5}{2}}}{2 \sqrt{2} \delta^{2}}\right)^{q}\left(2^{L} \sqrt{L+1}\right)\left(1+(\omega h)^{q-K+j+4}\right) \mathrm{e}^{\omega h} \\
& \cdot h^{K+1-j} \frac{1}{(q+1)^{\frac{q+1}{2}}} \omega\left\|V_{2}[Q]\right\| \|_{K, \omega, D} \\
\stackrel{(41)}{\leq} & C \mathrm{e}^{\left(1+\frac{3}{4}(1-\rho)\right) \omega h}\left(1+(\omega h)^{q-K+j+8}\right) h^{K+1-j} \\
& \cdot\left\{\left(\frac{\log (L+2)}{L+2}\right)^{\lambda(K+1-j)}+\frac{2^{L}}{\rho^{L-K+1}} \sqrt{\frac{L+1}{q+1}}\left(\frac{\mathrm{e}^{\frac{5}{2}}}{2 \sqrt{2} \delta^{2} \sqrt{q+1}}\right)^{q}\right\}\|u\|_{K+1, \omega, D},
\end{aligned}
$$

where the constant $C>0$ only depends on $\mathrm{j}, \mathrm{K}$ and the shape of $D$. If $K=j=0$, we have to use (5) instead of (6) in (41), so that (41) becomes

$$
\left\|V_{2}[Q]\right\|_{0, D} \leq C\left(1+(\omega h)^{4}\right) \mathrm{e}^{\frac{1}{2}(1-\rho) \omega h}\left(\|u\|_{0, D}+h|u|_{1, D}\right) .
$$

The rest of the proof continues as in the case $K \geq 1$ until the last but one step. For the last step, since

$$
\begin{aligned}
\omega\left\|V_{2}[Q]\right\|_{0, D} & \leq C\left(1+(\omega h)^{4}\right) \mathrm{e}^{\frac{1}{2}(1-\rho) \omega h} \omega\left(\|u\|_{0, D}+h|u|_{1, D}\right) \\
& \leq C\left(1+(\omega h)^{4}\right) \mathrm{e}^{\frac{1}{2}(1-\rho) \omega h}(1+\omega h)\|u\|_{1, \omega, D} \\
& \leq C\left(1+(\omega h)^{4}\right) \mathrm{e}^{\frac{3}{4}(1-\rho) \omega h}\|u\|_{1, \omega, D},
\end{aligned}
$$

we get exactly the same conclusion as in the case $K \geq 1$.

Theorem 5.3. (hp-estimates, $\mathrm{N}=3$ ) Let $u \in H^{K+1}(D)$ be a solution of the homogeneous Helmholtz equation in a domain $D \subset \mathbb{R}^{3}$ satisfying Assumption 2.1. Fix $q \geq 1$, set $p=(q+1)^{2}$ and let the directions $\left\{\boldsymbol{d}_{l, m}\right\}_{0 \leq|m| \leq l \leq q} \subset \mathbb{S}^{2}$ be such that the matrix $\boldsymbol{M}$ defined by (16) is invertible.

Then, for every integer $L$ satisfying

$$
0 \leq K \leq L \leq q, \quad L-K \leq\left\lfloor\frac{q-1}{2}\right\rfloor, \quad L \geq 2^{1 / \lambda},
$$


where $\lambda>0$ is the constant that depends only on the shape of $D$ from Theorem 3.2 , item (ii), there exists $\vec{\alpha} \in \mathbb{C}^{p}$ such that, for every $0 \leq j \leq K$,

$$
\begin{aligned}
\left\|u-\sum_{0 \leq|m| \leq l \leq q} \alpha_{l, m} \mathrm{e}^{i \omega \boldsymbol{x} \cdot \boldsymbol{d}_{l, m}}\right\|_{j, \omega, D} \leq & C\left(1+(\omega h)^{q+j-K+8}\right) \mathrm{e}^{\left(\frac{7}{4}-\frac{3}{4} \rho\right) \omega h} h^{K+1-j} \\
& \cdot\left\{L^{-\lambda(K+1-j)}+\frac{(L+1)^{2}\left\|\boldsymbol{M}^{-1}\right\|_{1}}{(\sqrt{2} \rho)^{L-K} q^{\frac{q+1}{2}}}\right\}\|u\|_{K+1, \omega, D},
\end{aligned}
$$

where the constant $C>0$ depends only on $j, K$ and the shape of $D$, but is independent of $q, L, \omega, h, u$ and the directions.

Proof. Let $Q$ be the generalized harmonic polynomial of degree at most $L$ equal to $Q_{L}^{\prime \prime}$ from Theorem 3.2, item (ii).

We proceed as we did in two-dimensions: for $K \geq 1$,

$$
\begin{aligned}
\left\|V_{2}[Q]\right\|_{K, \omega, D} & \leq\left\|V_{2}[u]\right\|_{K, \omega, D}+\left\|V_{2}[u]-V_{2}[Q]\right\|_{K, \omega, D} \\
& \stackrel{(55)}{\leq}(1+C)\left\|V_{2}[u]\right\|_{K, \omega, D} \\
& \stackrel{(6)}{\leq} C\left(1+(\omega h)^{4}\right) \mathrm{e}^{\frac{3}{4}(1-\rho) \omega h}\|u\|_{K, \omega, D},
\end{aligned}
$$

where $C$ depends only on $K$ and the shape of $D$.

$$
\begin{aligned}
& \left\|u-\sum_{0 \leq|m| \leq l \leq q} \alpha_{l, m} \mathrm{e}^{i \omega \boldsymbol{x} \cdot \boldsymbol{d}_{l, m}}\right\|_{j, \omega, D} \\
& \leq\|u-Q\|_{j, \omega, D}+\left\|Q-\sum_{0 \leq|m| \leq l \leq q} \alpha_{l, m} \mathrm{e}^{i \omega \boldsymbol{x} \cdot \boldsymbol{d}_{l, m}}\right\|_{j, \omega, D} \\
& \stackrel{(11),(39)}{\leq} C\left(1+(\omega h)^{j+6}\right) \mathrm{e}^{\frac{3}{4}(1-\rho) \omega h} L^{-\lambda(K+1-j)} h^{K+1-j}\|u\|_{K+1, \omega, D} \\
& +C\left(1+(\omega h)^{j+4}\right) \mathrm{e}^{\frac{1}{2} \omega h} h^{\frac{3}{2}-j}\left\|Q-\sum_{0 \leq|m| \leq l \leq q} \alpha_{l, m} \mathrm{e}^{i \omega \boldsymbol{x} \cdot \boldsymbol{d}_{l, m}}\right\|_{L^{\infty}\left(B_{h}\right)} \\
& \stackrel{(32), R=h}{\leq} C\left(1+(\omega h)^{j+6}\right) \mathrm{e}^{\frac{3}{4}(1-\rho) \omega h} L^{-\lambda(K+1-j)} h^{K+1-j}\|u\|_{K+1, \omega, D} \\
& +C \rho^{-L+K}\left(1+(\omega h)^{q+j-K+4}\right) \mathrm{e}^{\omega h} h^{K+1-j} \frac{(L+1)^{2}\left\|M^{-1}\right\|_{1}}{\sqrt{2}^{L} q^{\frac{q-3}{2}}(q+1)^{2}}\left\|V_{2}[Q]\right\|_{K, \omega, D} \\
& \stackrel{(43)}{\leq} C\left(1+(\omega h)^{q+j-K+8}\right) \mathrm{e}^{\left(1+\frac{3}{4}(1-\rho)\right) \omega h} h^{K+1-j} \\
& \cdot\left\{L^{-\lambda(K+1-j)}+\frac{(L+1)^{2}\left\|M^{-1}\right\|_{1}}{\rho^{L-K} \sqrt{2}^{L} q^{\frac{q+1}{2}}}\right\}\|u\|_{K+1, \omega, D},
\end{aligned}
$$

where $C>0$ only depends on $j, K$ and the shape of $D$. 
If $K=j=0,(43)$ becomes

$$
\begin{aligned}
\omega\left\|V_{2}[Q]\right\|_{0, D} & \leq \omega\left\|V_{2}[u]\right\|_{0, D}+\omega\left\|V_{2}[u]-V_{2}[Q]\right\|_{0, D} \\
(55), & j=k=0 \\
& \leq \omega\left\|V_{2}[u]\right\|_{0, D}+\omega C h\left|V_{2}[u]\right|_{1, D} \\
& \leq C(1+\omega h)\left\|V_{2}[u]\right\|_{1, \omega, D} \\
& \stackrel{(6)}{\leq} C\left(1+(\omega h)^{5}\right) \mathrm{e}^{\frac{3}{4}(1-\rho) \omega h}\|u\|_{1, \omega, D}
\end{aligned}
$$

where the constant $C$ depends only on the shape of $D$. We continue as before:

$$
\begin{aligned}
& \left\|u-\sum_{0 \leq|m| \leq l \leq q} \alpha_{l, m} \mathrm{e}^{i \omega \boldsymbol{x} \cdot \boldsymbol{d}_{l, m}}\right\|_{0, D} \leq\|u-Q\|_{0, D}+\left\|Q-\sum_{0 \leq|m| \leq l \leq q} \alpha_{l, m} \mathrm{e}^{i \omega \boldsymbol{x} \cdot \boldsymbol{d}_{l, m}}\right\|_{0, D} \\
& \stackrel{(11), k=j=0}{\leq} C\left(1+(\omega h)^{6}\right) \mathrm{e}^{\frac{3}{4}(1-\rho) \omega h} L^{-\lambda} h\|u\|_{1, \omega, D}+|D|^{\frac{1}{2}}\left\|Q-\sum_{0 \leq|m| \leq l \leq q} \alpha_{l, m} \mathrm{e}^{i \omega \boldsymbol{x} \cdot \boldsymbol{d}_{l, m}}\right\|_{L^{\infty}\left(B_{h}\right)} \\
& \stackrel{(32), R=h, K=0}{\leq} C\left(1+(\omega h)^{6}\right) \mathrm{e}^{\frac{3}{4}(1-\rho) \omega h} L^{-\lambda} h\|u\|_{1, \omega, D} \\
& +C \rho^{-L}\left(1+(\omega h)^{q}\right) \mathrm{e}^{\frac{1}{2} \omega h} \frac{|D|^{\frac{1}{2}}}{h^{\frac{3}{2}}} \frac{(L+1)^{2}\left\|M^{-1}\right\|_{1}}{\sqrt{2}^{L} q^{\frac{q-3}{2}}(q+1)^{2}} h \omega V_{2}[Q] \|_{0, D} \\
& \stackrel{(44)}{\leq} C\left(1+(\omega h)^{q+5}\right) \mathrm{e}^{\left(\frac{1}{2}+\frac{3}{4}(1-\rho)\right) \omega h} h\left\{L^{-\lambda}+\frac{(L+1)^{2}\left\|M^{-1}\right\|_{1}}{(\sqrt{2} \rho)^{L} q^{\frac{q+1}{2}}}\right\}\|u\|_{1, \omega, D},
\end{aligned}
$$

where $C>0$ only depends on the shape of $D$; this estimate completes the assertion of the theorem.

Remark 5.4. If the directions $\left\{\boldsymbol{d}_{l, m}\right\}_{0 \leq|m| \leq l \leq q} \subset \mathbb{S}^{2}$ in Theorem 5.3 are chosen as in Lemma 4.5, using the bound (38) of Corollary 4.8, instead of (32), the estimate (42) becomes

$$
\begin{aligned}
\left\|u-\sum_{0 \leq|m| \leq l \leq q} \alpha_{l, m} \mathrm{e}^{i \omega \boldsymbol{x} \cdot \boldsymbol{d}_{l, m}}\right\|_{j, \omega, D} \leq & C\left(1+(\omega h)^{q+j-K+8}\right) \mathrm{e}^{\left(\frac{7}{4}-\frac{3}{4}(1-\rho)\right) \omega h} h^{K+1-j} \\
& \cdot\left\{L^{-\lambda(K+1-j)}+\frac{(L+1)^{2}}{(\sqrt{2} \rho)^{L-K} q^{\frac{q-3}{2}}}\right\}\|u\|_{K+1, \omega, D},
\end{aligned}
$$

with $C>0$ depending only on $j, K$ and the shape of $D$, but independent of $q, L, \omega, h$ and $u$.

For $q \geq 2 K+1$, we can rewrite the error bounds of the two previous theorems in a simpler fashion.

Corollary 5.5. Let $u \in H^{K+1}(D)$ be a solution of the homogeneous Helmholtz equation and fix

$$
q \geq 2 K+1
$$

We consider the same assumptions on the domain $D$ and on the directions $\left\{\boldsymbol{d}_{k}\right\}_{k=1, \ldots, p}$ (in $3 \mathrm{D}$, we relabel the directions $\left\{\boldsymbol{d}_{l, m}\right\}$ as $\left.\left\{\boldsymbol{d}_{k}\right\}_{k=1, \ldots, p}\right)$ as in Theorems 5.2 and 5.3 for $N=2$ and $N=3$, respectively. In the three-dimensional case, we assume also $q \geq 2\left(1+2^{\frac{1}{\lambda}}\right)$, where $\lambda>0$ is the constant that depends only on the shape of $D$ from Theorem 3.2, item (ii). 
Then, there exists $\vec{\alpha} \in \mathbb{C}^{p}$ such that, for every $0 \leq j \leq K$,

$$
\begin{aligned}
& \left\|u-\sum_{k=1}^{p} \alpha_{k} \mathrm{e}^{i \omega \boldsymbol{x} \cdot \boldsymbol{d}_{k}}\right\|_{j, \omega, D} \leq C\left(1+(\omega h)^{q+j-K+8}\right) \mathrm{e}^{\left(\frac{7}{4}-\frac{3}{4} \rho\right) \omega h} h^{K+1-j} \\
& \begin{cases}{\left[\left(\frac{2 \log (q+2)}{q+2}\right)^{\lambda(K+1-j)}+\left(c_{0}(q+1)\right)^{-\frac{q}{2}}\right]\|u\|_{K+1, \omega, D},} & D \subset \mathbb{R}^{2}, \\
{\left[\left(\frac{q-2}{2}\right)^{-\lambda(K+1-j)}+(\sqrt{2} \rho q)^{-\frac{q-3}{2}}\left\|M^{-1}\right\|_{1}\right]\|u\|_{K+1, \omega, D},} & D \subset \mathbb{R}^{3},\end{cases}
\end{aligned}
$$

where $C>0$ depends only on $j, K$ and the shape of $D$, and in two-dimensions,

$$
c_{0}= \begin{cases}4 \mathrm{e}^{-5} \rho \delta^{4} & \text { general }\left\{\boldsymbol{d}_{k}\right\} \text { as in }(18), \\ 4 \mathrm{e}^{-1} \rho & \text { uniformly spaced }\left\{\boldsymbol{d}_{k}\right\} .\end{cases}
$$

Proof. Choose $L=\left\lfloor\frac{q-1}{2}\right\rfloor$ in Theorems 5.2 and 5.3 and see [12, Remark 3.1.4] for the uniformly spaced case in two-dimensions.

If we do not care about the dependence on $p$, in order to obtain a $h$-estimate with optimal order it is enough to require $q \geq K$ and, in three-dimensions, to assume $M$ invertible. This gives

$$
\left\|u-\sum_{k=1}^{p} \alpha_{k} \mathrm{e}^{i \omega \boldsymbol{x} \cdot \boldsymbol{d}_{k}}\right\|_{j, \omega, D} \leq C\left(1+(\omega h)^{q+j-K+8}\right) \mathrm{e}^{\left(\frac{7}{4}-\frac{3}{4} \rho\right) \omega h} h^{K+1-j}\|u\|_{K+1, \omega, D},
$$

where the constant $C$ does not depend on $h, \omega$ and $u$. No requirement depending on $\lambda$ is needed, because we can simply use the Bramble Hilbert theorem instead of Theorem A.3; see [12, Theorem 3.2.2].

Remark 5.6. The estimates in Corollary 5.5 look very similar in two and in three spatial dimensions, but few important differences must be pointed out.

If $D \subset \mathbb{R}^{2}$, any choice of (different) directions $\boldsymbol{d}_{k}$ guarantees the estimate and the convergence. The parameter $\lambda$, which provides the actual rate of convergence, can be computed explicitly by "measuring" the reentrant corners of $D$.

If $D \subset \mathbb{R}^{3}$, the estimate, as it is stated, which is valid provided that $M$ is invertible, guarantees the convergence in $q$ only if the growth of the norm of $\boldsymbol{M}^{-1}$ is controlled. This is true, for instance, for the optimal set of directions introduced in Lemma 4.5 and for Sloan's directions. Moreover, the rate $\lambda$ is not known. If a generalized harmonic polynomial approximation estimate like (11) with explicit order was available, then we could plug this coefficient in place of $\lambda$ in (45).

Remark 5.7. If $N=3$, assume that the norm of $M^{-1}$ is controlled (see Remark 5.6). The second term within the square brackets in the estimates of Corollary 5.5 converges to zero faster than exponentially, while the first one only algebraically. This gives the algebraic convergence of the best approximation, if $u$ has limited Sobolev regularity in $D$. On the other hand, the order of convergence of these estimates is given by the harmonic approximation problem described in Sect. 3. Thus, if the function $u$ is solution of the homogeneous Helmholtz equation in a domain $D^{\prime}$ such that $D \subset D^{\prime}, d\left(D, \partial D^{\prime}\right)=\delta>0$, we will have exponential convergence in $D$ (recall Remark 3.3). The speed will depend on $\delta$; see [16, Corollary 2.7] (2D) and [2] (3D).

\section{Appendix A. Proof of Theorem 3.2, part (ii)}

The fundamental approximation result by harmonic polynomials in more than two space dimensions is Theorem 1 of [2]. Assumption 2.1 guarantees that the hypotheses of this theorem are verified; see [12, Remark 2.1.6]. 
Theorem A.1. ([2, Theorem 1]) Let $D \subset \mathbb{R}^{N}$ satisfy Assumption 2.1. Then, there exist constants $p>0$, $b>1, q>0$ and $C>0$ depending only on $D$, such that, for every $\delta \in(0,1)$, for every $\phi$ harmonic in

$$
D^{\delta}=\left\{\boldsymbol{x} \in \mathbb{R}^{N}: d(\boldsymbol{x}, D)<\delta h\right\}=D+B_{\delta h}
$$

and for every integer $L>0$, there exists a harmonic polynomial $P$ of degree at most $L$ such that

$$
\|\phi-P\|_{L^{\infty}(D)} \leq C(\delta h)^{-p} b^{-L(\delta h)^{q}}\|\phi\|_{L^{\infty}\left(D^{\delta}\right)} .
$$

We cannot expect that the function $\phi$ we want to approximate can be extended outside the domain $D$ because a singularity can be present on the boundary of $D$. In order to use Theorem A.1, we need to introduce a function $T \phi$ defined on a neighborhood of $D$ such that: (i) $T \phi$ has the same Sobolev regularity of $\phi$; (ii) $T \phi$ is harmonic; and (iii) $T \phi$ approximates $\phi$ in the relevant Sobolev norms. In the next lemma, we build a function that satisfies these requirements using a technique analogous to the one used in $[16$, Lemma 2.11].

Lemma A.2. Let $D \subset \mathbb{R}^{N}$ be a domain as in Assumption $2.1, \phi \in H^{k+1}(D), k \in \mathbb{N}, \epsilon \in(0,1 / 2)$. Denote by $D_{\epsilon} \supset D$ the enlarged domain

$$
D_{\epsilon}:=\frac{1}{1-\epsilon} D=\left(1+\frac{\epsilon}{1-\epsilon}\right) D,
$$

and by $T_{l}[\phi](x)$ the functions defined on $D_{\epsilon}$ by

$$
T_{l}[\phi](\boldsymbol{x}):= \begin{cases}\sum_{|\boldsymbol{\alpha}| \leq l} \frac{1}{\boldsymbol{\alpha} !} D^{\boldsymbol{\alpha}} \phi((1-\epsilon) \boldsymbol{x})(\epsilon \boldsymbol{x})^{\boldsymbol{\alpha}} & l=0, \ldots, k \\ 0 & l=-1 .\end{cases}
$$

Then,

(i)

$$
\rho_{0} h \epsilon \leq d\left(D, \partial D_{\epsilon}\right) \leq 2 h \epsilon ;
$$

(ii) there exist a constant $C_{N, k}$ independent of $\epsilon, D$ and $\phi$ such that

$$
\left\|T_{k}[\phi]\right\|_{0, D_{\epsilon}} \leq C_{N, k} \sum_{l=0}^{k}(\epsilon h)^{l}|\phi|_{l, D}
$$

(iii) for every multi-index $\boldsymbol{\beta},|\boldsymbol{\beta}| \leq k+1$,

$$
D^{\boldsymbol{\beta}} T_{k}[\phi]=\sum_{l=0}^{|\boldsymbol{\beta}|}\left(\begin{array}{c}
|\boldsymbol{\beta}| \\
l
\end{array}\right) \epsilon^{l}(1-\epsilon)^{|\boldsymbol{\beta}|-l} T_{k-l}\left[D^{\boldsymbol{\beta}} \phi\right],
$$

which also implies that if $\phi$ is harmonic in $D$ then $T_{k}[\phi]$ is harmonic in $D_{\epsilon}$;

(iv) if $\phi$ is harmonic in $D$, there exist a constant $C_{N, k}$ independent of $\epsilon, D$ and $\phi$ such that

$$
\left|\phi-T_{k}[\phi]\right|_{j, D} \leq C_{N, k} \rho_{0}^{-j}(\epsilon h)^{k+1-j}|\phi|_{k+1, D} \quad \forall j=0, \ldots, k+1 .
$$

Proof. The bounds in (i) follow from the bounds

$$
\rho_{0} h \epsilon \leq \frac{\rho_{0} h \epsilon}{1-\epsilon} \leq d\left(D, \partial D_{\epsilon}\right) \leq \sup _{\boldsymbol{x} \in D} d\left(\boldsymbol{x}, \frac{1}{1-\epsilon} \boldsymbol{x}\right) \leq h\left(\frac{1}{1-\epsilon}-1\right)=\frac{h \epsilon}{1-\epsilon} \leq 2 h \epsilon,
$$

where the second inequality is proved in [15, Appendix A.3] (due to the slightly different definitions of $D_{\epsilon}$, the $\epsilon$ of [15, Appendix A.3] corresponds to our $\left.\frac{\epsilon}{1-\epsilon}\right)$. 
The bound (50) in (ii) is straightforward:

$$
\begin{aligned}
& \left\|T_{k}[\phi]\right\|_{0, D_{\epsilon}}^{2} \leq \int_{D_{\epsilon}} \sum_{|\boldsymbol{\alpha}| \leq k} \frac{1}{(\boldsymbol{\alpha} !)^{2}}\left|D^{\boldsymbol{\alpha}} \phi((1-\epsilon) \boldsymbol{x})\right|^{2}|\epsilon \boldsymbol{x}|^{2|\boldsymbol{\alpha}|} \mathrm{d} \boldsymbol{x}(\#\{\boldsymbol{\alpha}:|\boldsymbol{\alpha}| \leq k\}) \\
& \stackrel{\boldsymbol{y}=(1-\epsilon) \boldsymbol{x}}{\leq} \int_{D} \sum_{|\boldsymbol{\alpha}| \leq k} \frac{1}{(\boldsymbol{\alpha} !)^{2}}\left|D^{\boldsymbol{\alpha}} \phi(\boldsymbol{y})\right|^{2}\left|\frac{\epsilon h}{1-\epsilon}\right|^{2|\boldsymbol{\alpha}|} \frac{\mathrm{d} \boldsymbol{y}}{(1-\epsilon)^{N}}(\#\{\boldsymbol{\alpha}:|\boldsymbol{\alpha}| \leq k\}) \\
& \stackrel{l=|\boldsymbol{\alpha}|}{\leq} C_{N, k} \sum_{l=0}^{k}(\epsilon h)^{2 l}|\phi|_{l, D}^{2}
\end{aligned}
$$

For (iii), we proceed by induction on $|\boldsymbol{\beta}|$. For the case $|\boldsymbol{\beta}|=1, k>0$, given $m \in\{1, \ldots, N\}$, we set

$$
\boldsymbol{e}_{m}=(\underbrace{0, \ldots, 0}_{m-1}, 1,0, \ldots, 0) \in \mathbb{N}^{N}
$$

and denote by $\boldsymbol{\alpha}_{m}$ the $m$ th component of $\boldsymbol{\alpha}$; then,

$$
\begin{aligned}
& D_{\boldsymbol{x}_{m}} T_{k}[\phi](x)= \sum_{|\boldsymbol{\alpha}| \leq k} \frac{(1-\epsilon)}{\boldsymbol{\alpha} !}\left(D_{\boldsymbol{x}_{m}} D^{\boldsymbol{\alpha}}\right) \phi((1-\epsilon) \boldsymbol{x})(\epsilon \boldsymbol{x})^{\boldsymbol{\alpha}} \\
&+\sum_{\substack{|\boldsymbol{\alpha}| \leq k \\
\boldsymbol{\alpha} m \geq 1}} \frac{1}{\boldsymbol{\alpha} !} D^{\boldsymbol{\alpha}} \phi((1-\epsilon) \boldsymbol{x}) \epsilon \boldsymbol{\alpha}_{m}(\epsilon \boldsymbol{x})^{\boldsymbol{\alpha}-\boldsymbol{e}_{m}} \\
& \stackrel{\boldsymbol{\gamma}=\boldsymbol{\alpha - \boldsymbol { e } _ { m }}}{=}(1-\epsilon) T_{k}\left[D_{x_{m}} \phi\right](\boldsymbol{x})+\sum_{|\boldsymbol{\gamma}| \leq k-1} \frac{\epsilon\left(\boldsymbol{\gamma}_{m}+1\right)}{\left(\boldsymbol{\gamma}_{m}+1\right) \boldsymbol{\gamma} !} D^{\boldsymbol{\gamma}+\boldsymbol{e}_{m}} \phi((1-\epsilon) \boldsymbol{x})(\epsilon \boldsymbol{x})^{\boldsymbol{\gamma}} \\
&=(1-\epsilon) T_{k}\left[D_{\boldsymbol{x}_{m}} \phi\right](\boldsymbol{x})+\epsilon T_{k-1}\left[D_{\boldsymbol{x}_{m}} \phi\right](\boldsymbol{x}) .
\end{aligned}
$$

The case $|\beta|=1, k=0$, is given by

$$
D_{\boldsymbol{x}_{m}} T_{0}[\phi](\boldsymbol{x})=D_{\boldsymbol{x}_{m}}(\phi((1-\epsilon) \boldsymbol{x}))=(1-\epsilon) D_{\boldsymbol{x}_{m}} \phi((1-\epsilon) \boldsymbol{x})=(1-\epsilon) T_{0}\left[D_{\boldsymbol{x}_{m}} \phi\right](\boldsymbol{x}) ;
$$

this proves (51) in the case $|\boldsymbol{\beta}|=1$. Now, we proceed by induction for $2 \leq|\boldsymbol{\beta}| \leq k+1$. Let assume that (51) holds for every multi-index $\boldsymbol{\gamma}$ such that $1 \leq|\boldsymbol{\gamma}|<|\boldsymbol{\beta}| \leq k+1$. Given $\boldsymbol{\beta}$, there exists $m \in\{1, \ldots, N\}$ and $\gamma \in \mathbb{N}^{N}$ such that $\boldsymbol{\beta}=\boldsymbol{\gamma}+\boldsymbol{e}_{m}$; then,

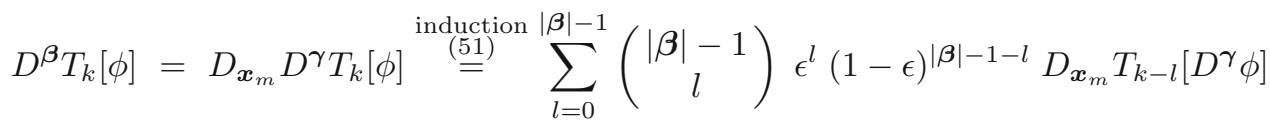

$$
\begin{aligned}
& \stackrel{(53)}{=} \sum_{l=0}^{|\boldsymbol{\beta}|-1}\left(\begin{array}{c}
|\boldsymbol{\beta}|-1 \\
l
\end{array}\right) \epsilon^{l}(1-\epsilon)^{|\boldsymbol{\beta}|-1-l}\left[(1-\epsilon) T_{k-l}\left[D^{\boldsymbol{\beta}} \phi\right]+\epsilon T_{k-l-1}\left[D^{\boldsymbol{\beta}} \phi\right]\right] \\
& =\sum_{l=0}^{|\boldsymbol{\beta}|}\left(\begin{array}{c}
|\boldsymbol{\beta}| \\
l
\end{array}\right) \epsilon^{l}(1-\epsilon)^{|\boldsymbol{\beta}|-l} T_{k-l}\left[D^{\boldsymbol{\beta}} \phi\right]
\end{aligned}
$$

where the last identity follows from Pascal's rule $\left(\begin{array}{c}j-1 \\ l\end{array}\right)+\left(\begin{array}{l}j-1 \\ l-1\end{array}\right)=\left(\begin{array}{l}j \\ l\end{array}\right)$.

In order to prove (52) of (iv), we write the Cauchy estimates for harmonic functions (see [18, eq. (36)])

$$
|\phi|_{j+k, \Omega} \leq C \nu^{-k}|\phi|_{j, \Omega+B_{\nu}} \quad \forall \phi \text { harmonic in } \Omega+B_{\nu}, j, k \in \mathbb{N}, \nu>0,
$$


for each open Lipschitz domain $\Omega \subset \mathbb{R}^{N}$. We fix a multi-index $\boldsymbol{\beta}$ and an integer $l, 0 \leq l \leq|\boldsymbol{\beta}|=j \leq k+1$. From the formula for the remainder of the multivariate Taylor polynomial, we have

$$
\begin{aligned}
& \left\|D^{\boldsymbol{\beta}} \phi-T_{k-l}\left[D^{\boldsymbol{\beta}} \phi\right]\right\|_{0, D}^{2} \\
& =\left.\left.\int_{D}\right|_{|\boldsymbol{\alpha}|=k-l+1} \frac{k-l+1}{\boldsymbol{\alpha} !}(\boldsymbol{x} \epsilon)^{\boldsymbol{\alpha}} \int_{0}^{1}(1-t)^{k-l} D^{\boldsymbol{\alpha}} D^{\boldsymbol{\beta}} \phi((1-\epsilon+t \epsilon) \boldsymbol{x}) \mathrm{d} t\right|^{2} \mathrm{~d} \boldsymbol{x} \\
& \leq C_{k, N}(h \epsilon)^{2(k-l+1)} \int_{0}^{1}(1-t)^{2(k-l)} \sum_{|\boldsymbol{\alpha}|=k-l+1} \int_{D}\left|D^{\boldsymbol{\alpha}} D^{\boldsymbol{\beta}} \phi((1-\epsilon+t \epsilon) \boldsymbol{x})\right|^{2} \mathrm{~d} \boldsymbol{x} \mathrm{d} t \\
& \leq C_{k, N}(h \epsilon)^{2(k-l+1)} \int_{0}^{1}(1-t)^{2(k-l)}|\phi|_{k-l+1+j,(1-\epsilon+t \epsilon) D}^{2} \mathrm{~d} t,
\end{aligned}
$$

where the seminorm on the right-hand side is well defined, though $\phi$ belongs only to $H^{k+1}(D)$, because since it is harmonic, it is $C^{\infty}$ in the interior of $D$. Thus,

$$
\begin{aligned}
& \left\|D^{\boldsymbol{\beta}} \phi-T_{k-l}\left[D^{\boldsymbol{\beta}} \phi\right]\right\|_{0, D}^{2} \\
& \stackrel{(54)}{\leq} C_{k, N}(h \epsilon)^{2(k-l+1)} \int_{0}^{1}(1-t)^{2(k-l)} d((1-\epsilon+t \epsilon) D, \partial D)^{-2(j-l)}|\phi|_{k+1, D}^{2} \mathrm{~d} t \\
& \quad \leq C_{k, N} \rho_{0}^{-2 j}(h \epsilon)^{2(k-j+1)}|\phi|_{k+1, D}^{2},
\end{aligned}
$$

because $(1-\epsilon+t \epsilon) D$ is star-shaped with respect to $B_{\rho_{0} h(1-\epsilon+t \epsilon)}, d((1-\epsilon+t \epsilon) D, \partial D) \geq \rho_{0} h(1-t) \epsilon$ thanks to [15, Appendix A.3], and the remaining integral is $\int_{0}^{1}(1-t)^{2(k-j)} \mathrm{d} t \leq 1$.

Finally, we use the fact that the sum of the coefficients in (51) is equal to 1 and obtain

$$
\begin{aligned}
\left|\phi-T_{k}[\phi]\right|_{j, D} & \leq \sum_{|\boldsymbol{\beta}|=j}\left\|D^{\boldsymbol{\beta}} \phi-D^{\boldsymbol{\beta}} T_{k}[\phi]\right\|_{0, D} \\
& \stackrel{(51)}{=} \sum_{|\boldsymbol{\beta}|=j}\left\|\sum_{l=0}^{j}\left(\begin{array}{l}
j \\
l
\end{array}\right) \epsilon^{l}(1-\epsilon)^{j-l}\left(D^{\boldsymbol{\beta}} \phi-T_{k-l}\left[D^{\boldsymbol{\beta}} \phi\right]\right)\right\|_{0, D} \\
& \leq \sum_{|\boldsymbol{\beta}|=j} \sum_{l=0}^{j}\left(\begin{array}{l}
j \\
l
\end{array}\right) \epsilon^{l}(1-\epsilon)^{j-l}\left\|D^{\boldsymbol{\beta}} \phi-T_{k-l}\left[D^{\boldsymbol{\beta}} \phi\right]\right\|_{0, D} \\
& \leq C_{k, N} \rho_{0}^{-j}(h \epsilon)^{k+1-j}|\phi|_{k+1, D} .
\end{aligned}
$$

This lemma allows to apply Theorem A.1 to harmonic functions with given Sobolev regularity in $D$, regardless of whether they can be extended outside this set. For $L$ large enough, the obtained order of convergence is algebraic and depends on the difference of the orders of the norms on the right- and left-hand sides (namely, $k+1-j$ ) and on a parameter $\lambda$ that depends on the geometry of the domain. Without any further assumption on $D$, we cannot expect to find an explicit value for $\lambda$. The following theorem is the three-dimensional analog of Theorem 2.9 of [16].

Theorem A.3. Fix $k \in \mathbb{N}$ and let $D \subset \mathbb{R}^{N}, N \geq 2$ be a domain as in Assumption 2.1. Then, there exist three constants:

$$
\begin{array}{ll}
C>0 & \text { depending only on } k, N \text { and the shape of } D, \\
q>0, b>1 & \text { depending only on } N \text { and the shape of } D
\end{array}
$$


such that

for every $L \geq \max \left\{k, 2^{q}\right\}$ and for every $\phi \in H^{k+1}(D)$ harmonic in $D$,

there exists a harmonic polynomial $P$ of degree $L$ that satisfies

$$
\begin{aligned}
& |\phi-P|_{j, D} \leq C h^{k+1-j}\left(L^{-\lambda(k+1-j)}+b^{-L^{1-\lambda q}} L^{\lambda\left(1+j+\frac{N}{2}\right)}\right)|\phi|_{k+1, D} \\
& \quad \forall 0 \leq j \leq k+1, \quad \forall \lambda \in(\log 2 / \log L, 1 / q) .
\end{aligned}
$$

If the degree $L$ is large enough, since $1-\lambda q$ is positive, the second term on the right-hand side is smaller than the first one and the convergence in $L$ is algebraic with order $\lambda(k+1-j)$. The coefficient $\lambda$ depends only on the shape of $D$ (through the constant $q$ of Theorem A.1).

Proof of Theorem A.3: Firstly, we fix three small positive constants $\epsilon_{1}, \epsilon_{2}, \epsilon_{3}$ in the interval $(0,1 / 2)$ and define $\epsilon_{*}:=1-\left(1-\epsilon_{1}\right)\left(1-\epsilon_{2}\right)\left(1-\epsilon_{3}\right)<\epsilon_{1}+\epsilon_{2}+\epsilon_{3}$. For every domain $\Omega$, we can define

$$
\begin{aligned}
& \hat{\Omega}:=\frac{1}{h} \Omega, \quad \Omega_{\epsilon}^{\prime}:=\frac{1}{1-\epsilon_{1}} \Omega, \quad \Omega_{\epsilon}^{\prime \prime}:=\frac{1}{1-\epsilon_{2}} \Omega_{\epsilon}^{\prime}=\frac{1}{\left(1-\epsilon_{1}\right)\left(1-\epsilon_{2}\right)} \Omega, \\
& \Omega_{\epsilon}^{\prime \prime \prime}:=\frac{1}{1-\epsilon_{3}} \Omega_{\epsilon}^{\prime \prime}=\frac{1}{\left(1-\epsilon_{1}\right)\left(1-\epsilon_{2}\right)\left(1-\epsilon_{3}\right)} \Omega=\frac{1}{1-\epsilon_{*}} \Omega .
\end{aligned}
$$

For every function $f$ defined on $\Omega$, we also define $\hat{f}(\hat{\boldsymbol{x}})=f(h \hat{\boldsymbol{x}})$ on $\hat{\Omega}$.

We apply Theorem A.1: for every $T \in H^{j}\left(D_{\epsilon}^{\prime \prime \prime}\right)$ harmonic, there exists a harmonic polynomial $\tilde{P}^{L}$ of degree at most $L$ such that

$$
\begin{aligned}
\left|T-\tilde{P}^{L}\right|_{j, D} & \leq C_{N, j} h^{\frac{N}{2}-j}\left|\hat{T}-\hat{\tilde{P}}^{L}\right|_{j, \hat{D}} \stackrel{(54),(49)}{\leq} C_{N, j} h^{\frac{N}{2}-j}\left(\rho_{0} \epsilon_{1}\right)^{-j}\left\|\hat{T}-\hat{\tilde{P}}^{L}\right\|_{0, \hat{D}_{\epsilon}^{\prime}} \\
& \leq C_{N, j} h^{\frac{N}{2}-j}\left|\hat{D}_{\epsilon}^{\prime}\right|^{\frac{1}{2}}\left(\rho_{0} \epsilon_{1}\right)^{-j}\left\|\hat{T}-\hat{\tilde{P}}^{L}\right\|_{L^{\infty}\left(\hat{D}_{\epsilon}^{\prime}\right)} \\
& \stackrel{(47)}{\leq} C_{N, j, \hat{D}} h^{\frac{N}{2}-j}\left(\frac{1}{1-\epsilon_{1}}\right)^{\frac{N}{2}}\left(\rho_{0} \epsilon_{1}\right)^{-j} \epsilon_{2}^{-p} b^{-L \epsilon_{2}^{q}}\|\hat{T}\|_{L^{\infty}\left(\hat{D}_{\epsilon}^{\prime \prime}\right)} \\
& \leq C_{N, j, \hat{D}} h^{\frac{N}{2}-j}\left(\rho_{0} \epsilon_{1}\right)^{-j} \epsilon_{2}^{-p} b^{-L \epsilon_{2}^{q}} \epsilon_{3}^{-\frac{N}{2}}\|\hat{T}\|_{0, \hat{D}_{\epsilon}^{\prime \prime \prime}} \\
& \leq C_{N, j, \hat{D}} h^{-j} \epsilon_{1}^{-j} \epsilon_{2}^{-p} b^{-L \epsilon_{2}^{q}} \epsilon_{3}^{-\frac{N}{2}}\|T\|_{0, D_{\epsilon}^{\prime \prime \prime}}
\end{aligned}
$$

where the bound in the second-last step follows from the mean value theorem for harmonic functions (see $[18$, eq. (33)]).

Now, we define

$$
\tilde{\phi}:=\phi-Q^{k+1} \phi
$$

where $Q^{k+1} \phi$ is the Taylor polynomial of $\phi$ (of degree $k$ ) averaged on $B_{\rho_{0} h}$ from Definition 4.1.3 of [3]. We choose

$$
T:=T_{k}[\tilde{\phi}]
$$

from Lemma A.2, using $\epsilon=\epsilon_{*}$. Let $\tilde{P}^{L}$ be the polynomial that approximate $T$ on $D$ from Theorem A.1 as above, so that (56) is satisfied. Finally, we define

$$
P^{L}:=\tilde{P}^{L}+Q^{k+1} \phi
$$

that is a harmonic polynomial of degree at most $L$, because $k \leq L$ and thanks to [3, Proposition 4.1.17] or $[12$, eq. (2.4)]. 
These definitions allow to gather all the approximation results proved so far in the following estimate:

$$
\begin{aligned}
\left|\phi-P^{L}\right|_{j, D} & =\left|\tilde{\phi}+Q^{k+1} \phi-\tilde{P}^{L}-Q^{k+1} \phi\right|_{j, D} \leq\left|\tilde{\phi}-T_{k}[\tilde{\phi}]\right|_{j, D}+\left|T_{k}[\tilde{\phi}]-\tilde{P}^{L}\right|_{j, D} \\
& \quad \leq C_{N, k} \rho_{0}^{-j}\left(\epsilon_{*} h\right)^{k+1-j}|\tilde{\phi}|_{k+1, D}+C_{N, j, \hat{D}} h^{-j} \frac{\epsilon_{1}^{-j} \epsilon_{2}^{-p} \epsilon_{3}^{-\frac{N}{2}}}{b^{L \epsilon_{2}^{q}}} \|\left. T_{k}[\tilde{\phi}]\right|_{0, D_{\epsilon}^{\prime \prime \prime}} \\
& \stackrel{(50)}{\leq} C_{N, j, k, \hat{D}}\left(\left(\epsilon_{*} h\right)^{k+1-j}|\tilde{\phi}|_{k+1, D}+\frac{\epsilon_{1}^{-j} \epsilon_{2}^{-p} \epsilon_{3}^{-\frac{N}{2}}}{b^{L \epsilon_{2}^{q}}} \sum_{l=0}^{k} \epsilon_{*}^{l} h^{l-j}|\tilde{\phi}|_{l, D}\right) \\
& \\
& \leq C_{N, j, k, \hat{D}}\left(\epsilon_{*}^{k+1-j}+\frac{\epsilon_{1}^{-j} \epsilon_{2}^{-p} \epsilon_{3}^{-\frac{N}{2}}}{b^{L \epsilon_{2}^{q}}} \sum_{l=0}^{k} \epsilon_{*}^{l}\right) h^{k+1-j}|\phi|_{k+1, D} \\
& \leq C_{N, j, k, \hat{D}}\left(\epsilon_{*}^{k+1-j}+\frac{\epsilon_{1}^{-j} \epsilon_{2}^{-p} \epsilon_{3}^{-\frac{N}{2}}}{b^{L \epsilon_{2}^{q}}}\right) h^{k+1-j}|\phi|_{k+1, D},
\end{aligned}
$$

as $Q^{k+1} \phi$ is a polynomial of degree at most $k$. Now, for every $\lambda \in(\log 2 / \log L, 1 / q)$, we can fix $\epsilon_{1}=\epsilon_{2}=$ $\epsilon_{3}=L^{-\lambda}<\frac{1}{2}$. This gives

$$
\left|\phi-P^{L}\right|_{j, D} \leq C_{N, j, k, \hat{D}}\left(L^{-\lambda(k+1-j)}+\frac{L^{\lambda\left(j+p+\frac{N}{2}\right)}}{b^{L^{1-\lambda q}}}\right) h^{k+1-j}|\phi|_{k+1, D},
$$

which completes the proof.

In order to prove the assertion of Theorem 3.2, it is enough to use the continuity of the Vekua operators to transfer the result of the previous theorem to the Helmholtz setting. We define $Q_{L}^{\prime \prime}=V_{1}[P]$, where $P$ is the approximating polynomial of $\phi=V_{2}[u]$ in Theorem A.3:

$$
\begin{aligned}
\left\|u-Q_{L}^{\prime \prime}\right\|_{j, \omega, D}^{2} & \stackrel{(4)}{\leq} C_{\hat{D}}(1+j)^{3 N+1} \mathrm{e}^{2 j}\left(1+(\omega h)^{2}\right)^{2} \sum_{l=0}^{j} \omega^{2(j-l)}\left|V_{2}[u]-P\right|_{l, D}^{2} \\
& \stackrel{(55)}{\leq} C_{j, k, \hat{D}}\left(1+(\omega h)^{2}\right)^{2} \sum_{l=0}^{j} \omega^{2(j-l)} h^{2(k+1-l)} L^{-2 \lambda(k+1-l)}\left|V_{2}[u]\right|_{k+1, D}^{2} \\
& \leq C_{j, k, \hat{D}}\left(1+(\omega h)^{j+2}\right)^{2} L^{-2 \lambda(k+1-j)} h^{2(k+1-j)}\left|V_{2}[u]\right|_{k+1, D}^{2} \\
& \stackrel{(6)}{\leq} C_{j, k, \hat{D}}\left(1+(\omega h)^{j+6}\right)^{2} \mathrm{e}^{\frac{3}{2}(1-\rho) \omega h} L^{-2 \lambda(k+1-j)} h^{2(k+1-j)}\|u\|_{k+1, \omega, D}^{2} .
\end{aligned}
$$

\section{References}

1. Babuška, I., Melenk, J.M.: The partition of unity method. Int. J. Numer. Methods Eng. 40, 727-758 (1997)

2. Bagby, T., Bos, L., Levenberg, N.: Quantitative approximation theorems for elliptic operators. J. Approx. Theory 85, 69-87 (1996)

3. Brenner, S.C., Scott, L.R.: Mathematical Theory of Finite Element Methods, 3rd edn., Texts in Applied Mathematics. Springer, New York (2007)

4. Buffa, A., Monk, P.: Error estimates for the ultra weak variational formulation of the Helmholtz equation. M2AN, Math. Model. Numer. Anal. 42, 925-940 (2008)

5. Cessenat, O., Després, B.: Application of an ultra weak variational formulation of elliptic PDEs to the two-dimensional Helmholtz equation. SIAM J. Numer. Anal. 35, 255-299 (1998)

6. Colton, D., Kress, R.: Inverse Acoustic and Electromagnetic Scattering Theory, vol. 93 of Applied Mathematical Sciences, 2nd edn. Springer, Heidelberg (1998)

7. Farhat, C., Harari, I., Franca, L.: The discontinuous enrichment method. Comput. Methods Appl. Mech. Eng. 190, 6455-6479 (2001)

8. Gautschi, W.: On inverses of Vandermonde and confluents Vandermonde determinants. Numer. Math. 4, 117-123 (1962) 
9. Gilbarg, D., Trudinger, N.: Elliptic Partial Differential Equations of Second Order, Classics in Mathematics, 2nd edn. Springer, Berlin (1983)

10. Gittelson, C.: Plane Wave Discontinuous Galerkin Methods. Master's thesis. SAM, ETH Zürich, Zürich, Switzerland (2008)

11. Gittelson, C.J., Hiptmair, R., Perugia, I.: Plane wave discontinuous Galerkin methods: analysis of the $h$-version. M2AN Math. Model. Numer. Anal. 43, 297-332 (2009)

12. Hiptmair, R., Moiola, A., Perugia, I.: Approximation by Plane Waves. Technical report 2009-27, SAM-ETH Zürich, ETH Zürich, Switzerland (2009)

13. Hiptmair, R., Moiola, A., Perugia, I.: Plane wave discontinuous Galerkin methods for the 2D Helmholtz equation: analysis of the $p$-version. SIAM J. Numer. Anal. 49, 264-284 (2011)

14. Lebedev, N.N.: Special Functions and Their Applications. Prentice-Hall, Englewood Cliffs, N.J. (1965)

15. Melenk, J.M.: On Generalized Finite Element Methods. PhD thesis, University of Maryland, Maryland (1995)

16. Melenk, J.M.: Operator adapted spectral element methods I: harmonic and generalized harmonic polynomials. Numer. Math. 84, 35-69 (1999)

17. Moiola, A.: Approximation Properties of Plane Wave Spaces and Application to the Analysis of the Plane Wave Discontinuous Galerkin Method. Technical report 2009-06, SAM-ETH Zürich, ETH Zürich, Switzerland (2009)

18. Moiola, A., Hiptmair, R., Perugia, I.: Vekua theory for the Helmholtz operator. Z. Angew. Math. Phys. (2011, to appear) 19. Müller, C.: Spherical Harmonics, vol. 17 of Lecture Notes in Mathematics. Springer, Berlin (1966)

20. Nédélec, J.-C.: Acoustic and Electromagnetic Equations: Integral Representations for Harmonic Problems, vol. 44 of Applied Mathematical Sciences. Springer, Berlin (2001)

21. Reimer, M.: Constructive theory of multivariate functions. Bibliographisches Institut, Mannheim (1990) With an application to tomography

22. Sloan, I.H., Womersley, R.S.: Extremal systems of points and numerical integration on the sphere. Adv. Comput. Math. 21, 107-125 (2004)

23. Vekua, I.N.: New Methods for Solving Elliptic Equations. North-Holland Publishing Co., Amsterdam (1967)

24. Womersley, R.S., Sloan, I.H.: Interpolation and Cubature on the Sphere. http://web.maths.unsw.edu.au/ rsw/Sphere/

A. Moiola and R. Hiptmair

SAM, ETH Zürich

8092 Zürich

Switzerland

e-mail: andrea.moiola@sam.math.ethz.ch

R. Hiptmair

e-mail: hiptmair@sam.math.ethz.ch

I. Perugia

Dipartimento di Matematica

Università di Pavia

Via Ferrata 1

27100 Pavia

Italy

e-mail: ilaria.perugia@unipv.it

(Received: August 18, 2010; revised: March 24, 2011) 